\title{
ON A SYSTEM OF NON-LINEAR ORDINARY DIFFERENTIAL EQUATIONS CONTAINING A PARAMETER
}

\author{
By Masahiro Iwano and Tosiya SaIto
}

1. Statement of the problem. In this note, we consider a system of $n$ equations of the type

$$
\varepsilon^{\sigma} \frac{d y_{j}}{d x}=\lambda_{j}(x, \varepsilon) y_{j}+\varepsilon^{\sigma} \sum_{k=1}^{n} a_{j k}(x, \varepsilon) y_{k}+\varepsilon^{\sigma} f_{j}\left(x, y_{1}, \cdots, y_{n}, \varepsilon\right), \quad j=1, \cdots, n,
$$

where we suppose that

1) $\sigma$ is a positive integer;

2) $\varepsilon$ is a complex parameter;

3) $x$ is a complex variable;

4) $\lambda_{j}(x, \varepsilon)$ are of the form

where

$$
\lambda_{j}(x, \varepsilon)=\sum_{h=0}^{\sigma-1} \lambda_{j}^{(h)}(x) \varepsilon^{h}, \quad j=1, \cdots, n,
$$

$$
\begin{array}{rlrl}
\lambda_{\jmath}{ }^{(h)}(x) & \equiv 0, & h=0,1, \cdots, \sigma-\sigma_{\jmath}-1 ; \\
\lambda_{\jmath}{ }^{\left(\sigma-\sigma_{j}\right)}(x) & \neq 0 ; & & \lambda_{\jmath}{ }^{\left(\sigma-\sigma_{j}\right)}(0) \neq 0 \quad \text { if } \quad \sigma_{\jmath}>0 ;
\end{array}
$$

and $\lambda_{j}{ }^{(h)}(x)$ are holomorphic for $|x|<r$;

5) $a_{j k}(x, \varepsilon)$ are holomorphic functions of $x$ and $\varepsilon$ for

$$
|x|<r, \quad \varepsilon \in \mathfrak{D}\left(\theta_{-}, \theta_{+}, \delta\right),
$$

where $\mathfrak{D}\left(\theta_{-}, \theta_{+}, \delta\right)$ denotes an angular domain

$$
\theta_{-}<\arg \varepsilon<\theta_{+}, \quad 0<|\varepsilon|<\delta ;
$$

6) $f_{j}\left(x, y_{1}, \cdots, y_{n}, \varepsilon\right)$ are of the form

$$
f_{j}\left(x, y_{1}, \cdots, y_{n}, \varepsilon\right)=\sum_{k_{1}+\cdots+k_{n} \geqq 2} f_{j k_{1} \cdots k_{n}}(x, \varepsilon) y_{1}{ }^{k_{1}} \cdots y_{n}{ }^{k_{n}}, \quad j=1, \cdots, n,
$$

where the power series in the right-hand members are uniformly convergent for

$$
|x|<r, \quad \max _{k}\left|y_{k}\right|<\eta, \quad \varepsilon \in \mathscr{D}\left(\theta_{-}, \theta_{+}, \delta\right),
$$

and the coefficients $f_{j k_{1} \cdots k_{n}}(x, \varepsilon)$ are holomorphic functions of $x$ and $\varepsilon$ for

$$
|x|<r, \quad \varepsilon \in \mathfrak{D}\left(\theta_{-}, \theta_{+}, \delta\right) ;
$$

7) when $\varepsilon$ tends to zero in $\mathfrak{D}\left(\theta_{-}, \theta_{+}, \delta\right), a_{j k}(x, \varepsilon)$ and $f_{j k_{1} \cdots k_{n}}(x, \varepsilon)$ admit asymptotic expansions in powers of $\varepsilon$ valid uniformly for $|x|<r$.

Our aim is to establish the existence of the solution of (1.1) containing several arbitrary constants and converging to 0 as $\varepsilon$ tends to 0 in $\mathfrak{D}\left(\theta_{-}, \theta_{+}, \delta\right)$.

Our proof is essentially similar to that given by one of the authors in his

Received September 1, 1960. 
recent paper [4].

2. Theorem of existence. We consider a slightly modified system

$$
\begin{aligned}
\varepsilon^{\sigma} \frac{d y_{j}}{d x}=\lambda_{j}(x, \varepsilon) y_{j}+\varepsilon^{\sigma}\left(a_{j}(x, \varepsilon)+\sum_{k=1}^{n} a_{j k}(x, \varepsilon) y_{k}\right. & \\
& \left.+f_{j}\left(x, y_{1}, \cdots, y_{n}, \varepsilon\right)\right), \quad j=1, \cdots, n,
\end{aligned}
$$

where $a_{j}(x, \varepsilon)$ are functions satisfying the conditions 5 ) and 7 ).

This section will be devoted to the proof of the following existence theorem.

THEOREM 1. If the system (2.1) possesses a formal solution

$$
y_{j} \approx \sum_{k=0}^{\infty} p_{j}^{(k)}(x) \varepsilon^{k}, \quad j=1, \cdots, n,
$$

such that the coefficients $p_{j}{ }^{(k)}(x)$ are holomorphic functions of $x$ for $|x|<r^{\prime}$ and $p_{j}(0)=0$, then there exists a solution $y_{j}=\varphi_{j}(x, \varepsilon)$ holomorphic in $(x, \varepsilon)$ for

$$
|x|<r^{\prime \prime}, \quad \varepsilon \in \mathfrak{D}\left(\theta_{-}{ }^{\prime \prime}, \theta_{+}{ }^{\prime \prime}, \delta\right),
$$

which admits an asymptotic expansion (2.2) (valid for $|x|<r^{\prime \prime}$ ) as $\varepsilon$ tends to 0 in $\mathfrak{D}\left(\theta_{-}{ }^{\prime \prime}, \theta_{+}{ }^{\prime \prime}, \delta\right)$ where $r^{\prime \prime}, \theta_{-}{ }^{\prime \prime}, \theta_{+}{ }^{\prime \prime}, \delta^{\prime \prime}$ are suitably chosen constants.

First we put

$$
y_{\jmath}=z_{\jmath}+Q_{j N}(x, \varepsilon), \quad Q_{j N}(x, \varepsilon)=\sum_{l=0}^{N+\sigma-1} p_{j}^{(l)}(x) \varepsilon^{l},
$$

then (2.1) will be transformed into

$$
\varepsilon^{\sigma} \frac{d z_{j}}{d x}=\lambda_{j}(x, \varepsilon) z_{\jmath}+\varepsilon^{\sigma} h_{j}\left(x, z_{1}, \cdots, z_{n}, \varepsilon\right), \quad j=1, \cdots, n,
$$

where

$$
\begin{aligned}
\varepsilon^{\sigma} h_{j}\left(x, z_{1}, \cdots, z_{n}, \varepsilon\right) \equiv \varepsilon^{\sigma} \sum_{k=1}^{n} a_{j k}(x, \varepsilon)\left(z_{k}+Q_{k N}(x, \varepsilon)\right)+\varepsilon^{\sigma} a_{j}(x, \varepsilon) \\
+\lambda_{j}(x, \varepsilon) Q_{j N}(x, \varepsilon)+\varepsilon^{\sigma} f_{j}\left(x, z+Q_{N}(x, \varepsilon)\right)-\varepsilon^{\sigma} \frac{d Q_{j N}(x, \varepsilon)}{d x} .
\end{aligned}
$$

Obviously, if the positive constants $\gamma_{0}{ }^{\prime}, \eta_{0}{ }^{\prime}, \delta_{0}{ }^{\prime}$ are suitably chosen, $h_{j}(x, z, \varepsilon)$ are holomorphic in $(x, z, \varepsilon)$ and satisfy the inequalities

$$
\left|h_{j}(x, z, \varepsilon)\right| \leqq A_{\jmath} \max _{k}\left|z_{k}\right|+B_{N}|\varepsilon|^{N}, \quad j=1, \cdots, n
$$

in the domain

$$
|x|<r_{0}{ }^{\prime}, \quad \max _{k}\left|z_{k}\right|<\eta_{0}{ }^{\prime}, \quad \varepsilon \in \mathscr{D}\left(\theta_{-}, \theta_{+}, \delta_{0}{ }^{\prime}\right),
$$

where $A_{\jmath}$ and $B_{N}$ are positive constants. Putting

(2.3) will be transformed into

$$
z_{\jmath}=u_{\jmath} \exp \Lambda_{j}(x, \varepsilon), \quad \Lambda_{j}(x, \varepsilon)=\int_{x_{j}{ }^{0}}^{x} \lambda_{j}(x, \varepsilon) \varepsilon^{-\sigma} d x,
$$

$$
\frac{d u_{j}}{d x}=h_{j}\left(x, u_{1} \exp \Lambda_{1}(x, \varepsilon), \cdots, u_{n} \exp \Lambda_{n}(x, \varepsilon), \varepsilon\right) \exp \left(-\Lambda_{j}(x, \varepsilon)\right) .
$$


Here the values of $x_{j}{ }^{0}$ and the paths of integration will be determined later. If

$$
\begin{cases}\sigma_{\jmath}=0, & j=n^{\prime}+1, \cdots, n^{\prime \prime}-1 \\ \sigma_{\jmath}>0, & \text { otherwise, }\end{cases}
$$

we may suppose, without loss of generality, that there exists a real number $\Omega$ $(0<\Omega<\pi / 2)$ such that

$$
\begin{aligned}
\Re \frac{\lambda_{1}{ }^{\left(\sigma-\sigma_{1}\right)}(0) e^{-i \Omega}}{\varepsilon^{\sigma_{1}}} \geqq \cdots & \geqq \Re \frac{\lambda_{n^{\prime}}{ }^{\left(\sigma-\sigma_{n^{\prime}}\right)}(0) e^{-i \Omega}}{\varepsilon^{\sigma_{n^{\prime \prime}}}} \geqq \tau>0>-\tau \\
& \geqq \Re \frac{\lambda_{n^{\prime \prime}}{ }^{\left(\sigma-\sigma_{\left.n^{\prime \prime}\right)}\right.}(0) e^{-i \Omega}}{\varepsilon^{\sigma_{n^{\prime \prime}}}} \geqq \cdots \geqq \Re \frac{\lambda_{n}{ }^{\left(\sigma-\sigma_{n}\right)}(0) e^{-i \Omega}}{\varepsilon^{\sigma_{n}}}
\end{aligned}
$$

for $\arg \varepsilon=\theta_{0}\left(\theta_{-}<\theta_{0}<\theta_{+}\right)$, where $\tau$ is a small positive number. Then we have

$$
\left|\omega_{j}-\Omega-\sigma_{j} \theta_{0}\right|>\frac{\pi}{2}\left(j \leqq n^{\prime}\right) ; \quad\left|\omega_{j}-\Omega-\sigma_{j} \theta_{0}-\pi\right|<\frac{\pi}{2}\left(j \geqq n^{\prime \prime}\right) ;
$$

where $\omega_{j}=\arg \lambda_{j}{ }^{\left(\sigma-\sigma_{j}\right)}(0)$. So, if $\varphi, a, \gamma(>0)$ are chosen sufficiently small, we have

$$
\max _{j}\left|\omega_{j}-(\Omega \pm \varphi)-\sigma_{\jmath}\left(\theta_{0} \pm a\right)\right| \leqq \frac{\pi}{2}-r, \quad j=1, \cdots, n^{\prime},
$$

and

$$
\max _{k}\left|\omega_{k}-(\Omega \pm \varphi)-\sigma_{k}\left(\theta_{0} \pm a\right)-\pi\right| \leqq \frac{\pi}{2}-r, \quad k=n^{\prime \prime}, \cdots, n .
$$

Denote by $E(\rho, \varphi, \Omega)$ an inner part of the lozenge whose four vertices are

$$
a^{(1)}=\rho e^{-i \Omega}, \quad a^{(2)}=i a^{(1)} \tan \varphi, \quad a^{(3)}=-a^{(1)}, \quad a^{(4)}=-a^{(2)},
$$

respectively. Then, for the proof of the theorem, it is sufficient to prove the existence of a solution $u_{j}(x, \varepsilon)$ of (2.5) such that

1) $u_{j}(x, \varepsilon)$ are holomorphic functions of $(x, \varepsilon)$ for

$$
x \in E(\rho, \varphi, \Omega), \quad \varepsilon \in \mathfrak{D}\left(\theta_{-}{ }^{\prime \prime}, \theta_{+}{ }^{\prime \prime}, \delta^{\prime \prime}\right), \quad \theta_{-}{ }^{\prime \prime}=\theta_{0}-a, \quad{\theta_{+}}^{\prime \prime}=\theta_{0}+a,
$$

2) $u_{j}\left(x_{\jmath}{ }^{0}, \varepsilon\right)=u_{j}{ }^{0}, \quad j=1, \cdots, n$,

3) $u_{\jmath}$ satisfy the following inequalities in (2.10) if $K_{N}>0$ is chosen sufficiently large:

$$
\left|u_{j}(x, \varepsilon)\right| \leqq K_{N}|\varepsilon|^{N} \exp \left(-\Re \Lambda_{j}(x, \varepsilon)\right) .
$$

Let $x_{0}$ be an arbitrary point in $E(\rho, \varphi, \Omega)$ and $\Gamma_{j x_{0}}$ be a segment joining $x_{j}{ }^{0}$ and $x_{0} . \quad \Gamma_{j x_{0}}$ is clearly contained in $E(\rho, \varphi, \Omega)$. Any point $x$ on this segment can be represented as

$$
x=x_{j}{ }^{0}+s e^{2 \dot{\varphi}}
$$

where $s$ is the length of the segment $\overline{x_{\jmath}{ }^{0} x}$ and $\psi=\arg \left(x^{0}-x_{\jmath}{ }^{0}\right)$. Now we shall prove the following

Lemma 1. Let $j$ be the number for which $\sigma_{\jmath}>0$, then, on $\Gamma_{j x_{0}}$, we have

$$
\frac{d}{d s} \exp \left(-\Re \Lambda_{j}(x, \varepsilon)\right) \geqq \frac{\left|\lambda_{j}{ }^{(\sigma-\sigma}{ }_{j}{ }^{\prime}(0)\right| \sin \gamma}{2|\varepsilon|^{\sigma}} \exp \left(-\Re \Lambda_{j}(x, \varepsilon)\right)
$$


for any $\varepsilon \in \mathfrak{D}\left(\theta_{-}{ }^{\prime \prime}, \theta_{+}{ }^{\prime \prime}, \delta^{\prime \prime}\right)$.

Proof. Since $\lambda_{j}{ }^{\left(\sigma-\sigma_{j}\right)}(0) \neq 0$, we have

$$
-\frac{d}{d x} \Lambda_{j}(x, \varepsilon)=-\frac{\lambda_{j} \frac{\left(\sigma-\sigma_{j}\right)}{\varepsilon^{\sigma}}(0)}{\varepsilon_{\jmath}}+O\left(\frac{x}{\varepsilon^{\sigma} \jmath}\right) .
$$

Hence

$$
\frac{d}{d s}\left[-\Re \Lambda_{j}(x, \varepsilon)\right]=-\Re\left[\frac{\lambda_{j}{ }^{\left(\sigma-\sigma_{j}\right)}(0) e^{\imath \psi}}{\varepsilon^{\sigma}{ }_{\jmath}}\right]+O\left(\frac{x}{\varepsilon^{\sigma}{ }^{\sigma}}\right) .
$$

If $j \leqq n^{\prime}$, we have, by (2.8),

$$
\varphi-\frac{\pi}{2}+\gamma \leqq \arg \lambda_{\jmath}{ }^{\left(\sigma-\sigma_{j}\right)}(0)-\Omega-\sigma_{\jmath} \arg \varepsilon \leqq-\varphi+\frac{\pi}{2}-\gamma .
$$

Since $-\varphi \leqq \psi-\pi+\Omega \leqq \varphi$, we obtain

$$
\left|\arg \left(\frac{\lambda_{j}{ }^{\left(\sigma-\sigma_{j}\right)}(0) e^{2 \psi}}{\varepsilon^{\sigma}{ }_{j}}\right)-\pi\right| \leqq \frac{\pi}{2}-\gamma
$$

whence follows that

$$
\frac{d}{d s}\left[-\Re \Lambda_{j}(x, \varepsilon)\right] \geqq \frac{\left|\lambda_{j}{ }^{\left(\sigma-\sigma_{j}\right)}(0)\right| \sin \gamma}{|\varepsilon|^{\sigma_{j}}}+O\left(\frac{x}{\varepsilon^{\sigma_{j}}}\right) \geqq \frac{\left|\lambda_{j}{ }^{\left(\sigma-\sigma_{j}\right)}(0)\right| \sin \gamma}{2|\varepsilon|^{\sigma_{j}}} .
$$

Multiplying $\exp \left(-\Re \Lambda_{j}\right)$ on both sides of the above inequality, we obtain (2.12).

For $j \geqq n^{\prime \prime}$, proof can be carried out quite similarly.

Let $\widetilde{F}$ be the family of the systems $\left\{u_{1}, \cdots, u_{n}\right\}$ of the functions $u_{j}(x, \varepsilon)$, holomorphic in $(x, \varepsilon)$ for (2.10) and satisfying the inequality (2.11) with $K_{N} \delta^{\prime \prime N}<\eta_{0}^{\prime}$.

Let $\mathfrak{I}$ be the mapping which maps the system $\left\{u_{1}, \cdots, u_{n}\right\}$ to the system $\left\{U_{1}, \cdots, U_{n}\right\}$ where $U_{\text {j }}$ are defined by

$$
U_{j}(x, \varepsilon)=u_{\jmath}^{0}+\int_{x_{j}{ }^{0}}^{x} h_{j}(x, u(x, \varepsilon) \exp \Lambda(x, \varepsilon), \varepsilon) \exp \left(-\Lambda_{j}(x, \varepsilon)\right) d x .
$$

Here the integration is to be carried out along the path $\Gamma_{j x_{0}}$ and $\left|u_{j}{ }^{0}\right|$ $\leqq\left(K_{N} / 2\right)|\varepsilon|^{N}$.

Since $\{0, \cdots, 0\} \in \mathfrak{F}, \mathfrak{F}$ is not empty, and moreover, $\mathfrak{F}$ is evidently closed, convex and normal. So, to complete the proof, we have only to show that:

$1^{\circ}$. $\mathfrak{I}(\mathfrak{F}) \subset \mathfrak{F}$

$2^{\circ}$. $\mathfrak{I}$ is a continuous mapping;

$3^{\circ}$. under the condition (2.11), the solution of (2.5) such that $u_{j}\left(x_{j}{ }^{0}, \varepsilon\right)$ $=u_{j}{ }^{0}$ is unique.

Indeed, by a well-known fixed-point theorem, we can conclude, from $1^{\circ}$ and $2^{\circ}$, that there exists an element of $\mathfrak{F}$ such that

$$
\left\{u_{1}, \cdots, u_{n}\right\}=\left\{U_{1}, \cdots, U_{n}\right\}
$$

which is clearly a solution of (2.5) with desired properties. We denote this solution by $u_{j N}(x, \varepsilon)$. Then

$$
S_{j N}(x, \varepsilon)=Q_{j N}(x, \varepsilon)+u_{j N}(x, \varepsilon) \exp \Lambda_{j}(x, \varepsilon)
$$


is the solution of (2.1) whose asymptotic expansion naturally coincides with (2.2). To show that $S_{j N}(x, \varepsilon)$ does not depend on $N$, it suffices to prove that

$$
u_{j N} \equiv\left\{Q_{j N^{\prime}}+u_{j N^{\prime}} \exp \Lambda_{\jmath}-Q_{j N}\right\} \exp \left(-\Lambda_{j}\right)
$$

which is an immediate consequence of $3^{\circ}$.

Proof of $1^{\circ}$. The integrand of (2.13) being bounded, it is clear that $U_{j}(x, \varepsilon)$ are holomorphic for (2.10). So we have only to prove the inequality (2.11). Now, by (2.4), we have

$$
\begin{aligned}
\mid \int_{x_{j}{ }^{0}}^{x} h_{j}\left((x, u(x, \varepsilon) \exp \Lambda(x, \varepsilon), \varepsilon\} \exp \left(-\Lambda_{j}(x, \varepsilon)\right) d x \mid\right. \\
\leqq \int_{0}^{s_{0}}\left(A_{j} K_{N}+B_{N}\right)|\varepsilon|^{N} \exp \left(-\Re \Lambda_{j}(x, \varepsilon)\right) d s,
\end{aligned}
$$

where $s_{0}$ is the length of the segment $\Gamma_{J_{0}}$. As $u_{\jmath}{ }^{0}$ have been so chosen that $\left|u_{\jmath}{ }^{0}\right|<\left(K_{N} / 2\right)|\varepsilon|^{N}$, (2.11) would follow if we could show

$$
\left.\left.\left|\int_{0}^{s_{0}}\left(A_{j} K_{N}+B_{N}\right)\right| \varepsilon\right|^{N} \exp \left(-\Re \Lambda_{j}(x, \varepsilon)\right) d s\left|\leqq \frac{K_{N}}{2}\right| \varepsilon\right|^{N} \exp \left(-\Re \Lambda_{j}(x, \varepsilon)\right) .
$$

For $\sigma_{\jmath}=0$, this inequality is reduced to

$$
\left(A_{j} K_{N}+B_{N}\right) s_{0} \leqq \frac{K_{N}}{2},
$$

since, in this case, we have $A_{j}(x, \varepsilon) \equiv 0$. As $s_{0} \leqq 2 \rho$, this inequality is implied by

$$
4_{i} \rho\left(A_{j} K_{N}+B_{N}\right) \leqq K_{N}
$$

which is always satisfied if $\rho$ is taken sufficiently small.

If $\sigma_{\jmath}>0$, it suffices to show

$$
\left(A_{j} K_{N}+B_{N}\right)|\varepsilon|^{N} \exp \left(-\Re \Lambda_{j}(x, \varepsilon)\right) \leqq \frac{K_{N}}{2} \frac{d}{d s}\left[|\varepsilon|^{N} \exp \left(-\Re \Lambda_{j}(x, \varepsilon)\right)\right]
$$

which follows immediately from Lemma 1 if, as is always possible, $\delta^{\prime \prime}$ and $K_{N}{ }^{-1}$ are chosen so small that we have

$$
4|\varepsilon|^{\sigma_{j}}\left(A_{j} K_{N}+B_{N}\right) \leqq\left|\lambda_{j}{ }^{\left(\sigma-\sigma_{i}\right)}(0)\right| \sin \gamma \cdot K_{N} .
$$

$2^{\circ}$ is almost evident since the integral in (2.13) converges absolutely and uniformly.

Proof of $3^{\circ}$. Assume the contrary, and let $u_{j N}(x, \varepsilon)$ and $v_{j N}(x, \varepsilon)$ be two solutions of (2.5). Then the difference $w_{j} \equiv u_{j N}-v_{j N}$ satisfies the differential inequality

$$
\left\{\begin{array}{l}
\frac{d\left|w_{j}\right|}{d s} \leqq A_{\jmath} \max _{k, \Gamma k x_{0}}\left|w_{k}(x, \varepsilon) \exp \Lambda_{k}(x, \varepsilon)\right| \exp \left(-\Re \Lambda_{j}(x, \varepsilon)\right), \\
w_{j}\left(x_{\jmath}{ }^{0}, \varepsilon\right)=0
\end{array}\right.
$$

Taking the inequality (2.12) into account, we integrate the above inequality along $\Gamma_{J x_{0}}$, and obtain 
where

$$
\left|w_{j}(x, \varepsilon) \exp \Lambda_{j}(x, \varepsilon)\right| \leqq a_{\jmath} \max _{k, \Gamma k x_{0}}\left|w_{k}(x, \varepsilon) \exp \Lambda_{k}(x, \varepsilon)\right|,
$$

$$
a_{\jmath}= \begin{cases}s_{0} A_{\jmath}, & \left(\sigma_{\jmath}=0\right), \\ \frac{2|\varepsilon|^{\sigma_{j}} A_{j}}{\left|\lambda_{\jmath}{ }^{\left(\sigma-\sigma_{j}\right)}(0)\right| \sin \gamma}, & \left(\sigma_{\jmath}>0\right) .\end{cases}
$$

So, if we choose $\rho$ and $\delta^{\prime \prime}$ sufficiently small, we have $a_{\jmath} \leqq 1 / 2$ and the above inequality can be replaced by

$$
\left|w_{j}(x, \varepsilon) \exp \Lambda_{\jmath}(x, \varepsilon)\right| \leqq \frac{1}{2} \max _{k, \Gamma k x_{0}}\left|w_{k}(x, \varepsilon) \exp \Lambda_{k}(x, \varepsilon)\right|
$$

which shows that $\left|w_{j}(x, \varepsilon) \exp \Lambda_{j}(x, \varepsilon)\right|$ must vanish identically.

Therefore we have $w_{j} \equiv 0$ which is the required result.

3. Reduction of the linear part. Here we assert that, without loss of generality, we may suppose the coefficients $a_{j k}(x, \varepsilon)$ in (1.1) satisfy the condition

$$
a_{j k}(x, \varepsilon) \equiv 0 \quad \text { if } \quad \lambda_{j k}{ }^{\left(\sigma-\sigma_{j k}\right)}(0) \neq 0 \quad \text { or } \quad \sigma_{j k}=0,
$$

where we used the notation

$$
\lambda_{j}(x, \varepsilon)-\lambda_{k}(x, \varepsilon) \equiv \lambda_{j k}(x, \varepsilon)=\lambda_{j k}{ }^{\left(\sigma-\sigma_{j k}\right)}(x) \varepsilon^{\sigma-\sigma_{j k}}+\lambda_{j k}{ }^{\left(\sigma-\sigma_{j k}+1\right)}(x) \varepsilon^{\sigma-\sigma_{j_{k}}+1}+\cdots .
$$

This assertion is based on the following

THEOREM 2. The system

$$
\varepsilon^{\sigma} \frac{d y_{j}}{d x}=\lambda_{j}(x, \varepsilon) y_{j}+\varepsilon^{\sigma} \sum_{k=1}^{n} a_{j k}(x, \varepsilon) y_{k}
$$

can be transformed into the system

$$
\varepsilon^{\sigma} \frac{d z_{j}}{d x}=\lambda_{j}(x, \varepsilon) z_{\jmath}+\varepsilon^{\sigma} \sum_{k=1}^{n} b_{j k}(x, \varepsilon) z_{k}
$$

with the property

$$
b_{j k}(x, \varepsilon) \equiv 0 \quad \text { if } \quad \lambda_{j k}{ }^{\left(\sigma-\sigma_{j k}\right)}(0) \neq 0 \quad \text { or } \quad \sigma_{j k}=0
$$

by a linear substitution

$$
y_{j}=z_{\jmath}+\varepsilon^{\sigma} \sum_{k=1}^{n} p_{j k}(x, \varepsilon) z_{k},
$$

where $p_{j k}(x, \varepsilon)$ are holomorphic in the domain

$$
|x|<r_{2}(<r), \quad \varepsilon \in \mathfrak{D}\left(\theta_{-}{ }^{\prime}, \theta_{+}{ }^{\prime}, \delta\right), \quad\left(\theta_{-}<\theta_{-}{ }^{\prime}<\theta_{+}{ }^{\prime}<\theta_{+}, 0<\delta_{1}<\delta\right) .^{1}{ }^{1}
$$

Proof. For (3.2) to be transformed into (3.3) by (3.4), we must have

$$
\varepsilon^{\sigma} \frac{d p_{j k}}{d x}=\lambda_{j k} p_{j k}+\varepsilon^{\sigma}\left(a_{j k}-b_{j k}\right)+\varepsilon^{\sigma} \sum_{h=1}^{n}\left[a_{j h} p_{h k}-p_{j h} b_{h k}\right] \text {. }
$$

1) Our proof is essentially similar to Sibuya [5]. 
We put here $b_{j k} \equiv 0$ if $\lambda_{j k}{ }^{\left(\sigma-\sigma_{j k}\right)}(0) \neq 0$ and $p_{j k} \equiv 0$ if $\lambda_{j k}^{\left(\sigma-\sigma_{j k}\right)}(0)=0$. Then (3.6) will be reduced to

$$
\begin{gathered}
\varepsilon^{\sigma} \frac{d p_{j k}}{d x}=\lambda_{j k} p_{j k}+\varepsilon^{\sigma} a_{j k}+\varepsilon^{\sigma} \sum_{\lambda h k 0 \neq 0} a_{j h} p_{h k}-\varepsilon^{\sigma} \sum_{\lambda_{j h 0} \neq 0, \lambda} \sum_{h k 0 \neq 0} p_{j h} b_{h k}, \quad \lambda_{j k 0} \neq 0, \\
0=\varepsilon^{\sigma}\left(a_{j k}-b_{j k}\right)+\varepsilon^{\sigma} \sum_{\lambda h k 0 \neq 0} a_{j h} p_{h k}, \quad \lambda_{j k 0}=0,
\end{gathered}
$$

where the notation $\lambda_{j k 0}=\lambda_{j k}^{\left(\sigma-\sigma_{j k}\right)}(0)$ is adopted for simplicity's sake.

From (3.8) we immediately have

$$
b_{j k}=a_{j k}+\sum_{\lambda h k 0 \neq 0} a_{j h} p_{h k}, \quad \lambda_{j k 0}=0 .
$$

Substituting the expression (3.9) into the right-hand member of (3.7), we have

$$
\begin{aligned}
\varepsilon^{\sigma} \frac{d p_{j k}}{d x}=\lambda_{j k} p_{j k} & +\varepsilon^{\sigma} a_{j k}+\varepsilon^{\sigma} \sum_{\lambda h k_{0} \neq 0} a_{j h} p_{h k} \\
& +\varepsilon^{\sigma} \sum_{\lambda k k_{0} \neq 0} p_{j h}\left[a_{h k}+\sum_{\lambda l k 0 \neq 0} a_{h l} p_{l k}\right], \quad \lambda_{j k 0} \neq 0 .
\end{aligned}
$$

By assumption, $a_{j k}(x, \varepsilon)$ admits an asymptotic expansion

$$
a_{j k}(x, \varepsilon) \approx \sum_{p=0}^{\infty} a_{j k}^{(p)}(x) \varepsilon^{p} .
$$

Now we put formally

$$
p_{j k}(x, \varepsilon) \approx \sum_{p=\sigma_{j k}}^{\infty} p_{j k}{ }^{(p)}(x) \varepsilon^{p},
$$

and substitute (3.11) and (3.12) into the equation (3.10). Comparing the terms of the same degree in $\varepsilon$ on both sides of the equation, we can determine the coefficients $p_{j k}^{(p)}$ step by step.

Suppose that $p_{j k}^{(p)}(x)(p<N)$ have been determined as holomorphic functions of $x$. Then the coefficients $p_{j k}^{(N)}(x)$ will be determined from

$$
\lambda_{j k}{ }^{\left(\sigma-\sigma_{j k}\right)}(0) p_{j k}{ }^{(N)}=Q_{j k}\left(p_{r s}{ }^{(h)}, \frac{d p_{r s}{ }^{(h)}}{d x}, a_{r s}{ }^{(m)}\right)
$$

where the right-hand member is a linear form of $a_{r s}{ }^{(m)}\left(0<m \leqq N-\sigma_{r s}\right)$ whose coefficients are polynomials of $p_{r s}^{(h)}$ and $d p_{r s}{ }^{(h)} / d x(0<h<N)$. Hence $Q_{j k}$ are known holomorphic functions of $x$. Since $\lambda_{j k}{ }^{\left(\sigma-\sigma \sigma_{k}\right)}(0) \neq 0, p_{j k}{ }^{(N)}(x)$ can be determined as a holomorphic function of $x$. Thus (3.10) admits a formal solution of the type (3.12).

Since the system (3.10) can be regarded as a special form of the system (2.1), the conclusion of Theorem 1 is applicable. Hence (3.10) has a holomorphic solution $p_{j k}(x, \varepsilon)$ whose asymptotic expansion coincides with (3.12). Therefore there exists a substitution (3.4) such that the transformed system is of the form (3.3) and $b_{j k} \equiv 0$ if $\lambda_{j k}^{\left(\sigma-\sigma_{j k}\right)}(0) \neq 0$.

This transformation having been done, we consider the equations of (3.3) for which $\sigma_{j k}=0$. As $\sigma_{j k}=0$ means $\lambda_{j k}(x, \varepsilon) \equiv 0$, we have either

$$
\left.\lambda_{j}(x, \varepsilon) \equiv \lambda_{k}(x, \varepsilon) \equiv 0 \quad \text { (i.e. } \quad \sigma_{\jmath}=\sigma_{k}=0\right)
$$


or

$$
\lambda_{j}(x, \varepsilon) \equiv \lambda_{k}(x, \varepsilon) \neq 0 .
$$

For the first case, (3.3) will be written as

$$
\frac{d z_{j}}{d x}=\sum_{\sigma_{k}=0} b_{j k}(x, \varepsilon) z_{k} \quad\left(\sigma_{j}=0\right) .
$$

It is well known that there exists a linear substitution with holomorphic coefficients

$$
z_{\jmath}=u_{\jmath}+\sum_{\sigma_{k}=0} Q_{j k}(x, \varepsilon) u_{k}
$$

which transforms the system (3.13) into

$$
\frac{d u_{j}}{d x}=0 \text {. }
$$

Next we consider the second case: $\lambda_{\jmath}(x, \varepsilon) \equiv \lambda_{k}(x, \varepsilon) \neq 0$.

Let

$$
\varepsilon^{\sigma} \frac{d z_{j}}{d x}=\lambda_{j}(x, \varepsilon) z_{\jmath}+\varepsilon^{\sigma} \sum_{\lambda_{k} \equiv \lambda_{\jmath}} b_{j k}(x, \varepsilon) z_{k}
$$

be the equations of (3.3) for $\lambda_{\jmath} \equiv \lambda_{k} \neq 0$. Putting

$$
z_{j}=u_{j} \exp \Lambda_{j}(x, \varepsilon), \quad \Lambda_{j}(x, \varepsilon)=\int \lambda_{j}(x, \varepsilon) \varepsilon^{-\sigma} d x,
$$

(3.15) will become

$$
\frac{d u_{j}}{d x}=\sum b_{j k}(x, \varepsilon) u_{k}
$$

which is of the same form as (3.13). Therefore we can find a linear substitution with holomorphic coefficients

$$
u_{\jmath}=v_{\jmath}+\sum Q_{j k}(x, \varepsilon) v_{k}
$$

such that (3.16) will be reduced to $d v_{\jmath} / d x=0$.

Consequently, by composing these transformations, i.e.

$$
y \rightarrow z \rightarrow u, \quad \text { or } \quad y \rightarrow z \rightarrow u \rightarrow v,
$$

we arrive at the required result.

4. Formal transformation. Having finished the reduction of the linear part of the system (1.1) in the preceding section, our next step is to transform its non-linear part into a possibly simple form. For that purpose, we begin with the utterly formal construction of such transformation. Discussions concerning its convergence will be postponed to the latter sections.

Let us consider a formal transformation

$$
y_{l} \approx u_{2}+\sum_{k_{1}+\cdots+k_{n} \geq 2} p_{j \mathfrak{k}}(x, \varepsilon) u_{1}^{k_{1}} \cdots u_{n}{ }^{k_{n}}
$$

where we used a symbol to represent the arrangement of $n$ non-negative 
integers $\left(k_{1}, \cdots, k_{n}\right)$. We impose upon the coefficients $p_{j \mathfrak{t}}$ following two conditions:

1) $p_{j \nVdash}(x, \varepsilon)$ are holomorphic functions of $(x, \varepsilon)$ in the domain

$$
|x|<r_{\mathfrak{H}}, \quad \varepsilon \in \mathscr{D}\left(\theta_{-\mathfrak{x}}, \theta_{+\mathfrak{x}}, \delta_{\mathfrak{E}}\right),
$$

where the constants $r_{\mathfrak{t}}, \theta_{-\mathfrak{t}}, \theta_{+\mathfrak{t}}, \delta_{\mathfrak{k}}$ may generally depend upon $\mathfrak{k}$;

2) every $p_{j \nVdash}(x, \varepsilon)$ admits an asymptotic expansion valid for $|x|<r_{\mathfrak{x}}$

$$
p_{j \mathfrak{k}}(x, \varepsilon) \approx \sum_{h=\sigma}^{\infty} p_{j \mathfrak{\sharp}}^{(h)}(x) \varepsilon^{h}
$$

as $\varepsilon$ tends to 0 in $\mathfrak{D}\left(\theta_{-\mathfrak{x}}, \theta_{+\mathfrak{x}}, \delta_{\mathfrak{k}}\right)$ where the symbol $\sigma_{j \mathfrak{k}}$ is defined by a following formula:

$$
\lambda_{j \mathfrak{k}}(x, \varepsilon) \equiv \lambda_{j}(x, \varepsilon)-\sum_{\nu=1}^{n} k_{\nu} \lambda_{\nu}(x, \varepsilon) \equiv \lambda_{j \mathfrak{k}}^{\left(\sigma-\sigma_{j \mathfrak{\sharp}}\right)}(x) \varepsilon^{\sigma-\sigma_{j \mathfrak{}}}+O\left(\varepsilon^{\sigma-\sigma_{j \mathfrak{\sharp}}+1}\right) .
$$

Applying (4.1) to the system (1.1), it will be transformed into a formal system

$$
\varepsilon^{\sigma} \frac{d u_{\jmath}}{d x} \approx \lambda_{j}(x, \varepsilon) u_{\jmath}+\varepsilon^{\sigma}\left[\sum_{k=1}^{n} a_{j k}(x, \varepsilon) u_{k}+\sum_{k_{1}+\cdots \cdot k_{n} \geqq 2} c_{j \nVdash}(x, \varepsilon) u_{1}^{k_{1}} \cdots u_{n}^{k_{n}}\right] .
$$

The aim of this section is to establish the following

THEOREM 3. There exists a formal transformation (4.1) such that $c_{j \mathfrak{}}(x, \varepsilon)$ are all holomorphic in $(x, \varepsilon)$ in the domain $(4.2)_{\mathfrak{f}}$ and

$$
c_{j \mathfrak{k}}(x, \varepsilon) \equiv 0 \quad \text { if } \quad \lambda_{j \mathfrak{k}}^{\left(\sigma-\sigma_{j \mathfrak{f}}\right)}(0) \neq 0 \quad \text { or } \quad \sigma_{j \mathfrak{k}}=0 .
$$

CoROLlary. The system (2.1) admits a formal solution of the type (2.2) if $\lambda_{\jmath}{ }^{\left(\sigma-\sigma_{\mathcal{H}^{*}}\right.}(0) \neq 0$ for $\sigma_{\jmath}>0$.

Proof. We define the order of arrangements $\left(j, k_{1}, \cdots, k_{n}\right)$ of the nonnegative integers $j, k_{1}, \cdots, k_{n}$ in the following way.

If

or

$$
k_{1}+\cdots+k_{n}<q_{1}+\cdots+q_{n}
$$

$$
k_{1}+\cdots+k_{n}=q_{1}+\cdots+q_{n} \text { and } k_{s}=q_{s}(i<s \leqq n), k_{\imath}<q_{i},
$$

we shall call that $\left(k_{1}, \cdots, k_{n}\right)$ precedes $\left(q_{1}, \cdots, q_{n}\right)$ and denote it by $\left(k_{1}, \cdots, k_{n}\right)$ $\prec\left(q_{1}, \cdots, q_{n}\right)$. Then we define that $\left(j, k_{1} \cdots, k_{n}\right)$ precedes $\left(k, q_{1} \cdots, q_{n}\right)$ or, in symbols,

$$
\left(j, k_{1}, \cdots, k_{n}\right) \prec\left(k, q_{1}, \cdots, q_{n}\right),
$$

if $\left(k_{1}, \cdots, k_{n}\right) \prec\left(q_{1}, \cdots, q_{n}\right)$, or $\left(k_{1}, \cdots, k_{n}\right)=\left(q_{1}, \cdots, q_{n}\right)$ and $j>k$.

Suppose that $p_{j \mathfrak{k}}(x, \varepsilon)$ and $c_{j \mathfrak{k}}(x, \varepsilon)$ have been determined for $k_{1}+\cdots+k_{n}$ $<N$. The inverse transformation of (4.1) be written as

$$
u_{\jmath} \approx y_{j}+\sum q_{j} y_{1}{ }^{k_{1}} \cdots y_{n}{ }^{k_{n}}
$$

where $q_{j \mathfrak{l}}=-p_{j \mathfrak{}}+Q_{j \mathfrak{l}}\left(p_{l \xi}\right), Q_{j}\left(p_{l \xi}\right)$ being polynomials of $p_{l \xi}\left(h_{1}+\cdots+h_{n}>N\right)$ of degree $\leqq 2 N-1$. Differentiating (4.4) term by term, and substituting (4.1) and 
(4.3) into it, we obtain a formal equality between formal power series of $u_{1}$, $\cdots, u_{n}$. Equating the coefficients of $u_{1}^{k_{1}} \cdots u_{n}{ }^{k_{n}}$ on both sides, we can easily derive the following relations:

$$
\begin{aligned}
& \varepsilon^{\sigma} \frac{d p_{j \mathfrak{k}}}{d x}=\lambda_{j \mathfrak{t}}(x, \varepsilon) p_{j \mathfrak{k}}+\varepsilon^{\sigma} \sum_{j h_{0}=0} a_{j h}(x, \varepsilon) p_{h \mathfrak{H}}-\varepsilon^{\sigma}\left(\sum_{h=1}^{n} k_{h} a_{h h}(x, \varepsilon)\right) p_{j \mathfrak{k}}
\end{aligned}
$$

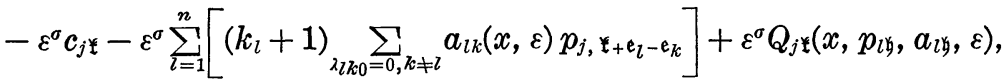

where

1) $\mathfrak{e}_{k}$ denotes $\left(\delta_{1 k}, \cdots, \delta_{n k}\right)\left(\delta_{j k}\right.$ : the Kronecker's symbol);

2) $\mathfrak{i}+\mathfrak{e}_{l}-\mathfrak{e}_{k}$ denotes $\left(k_{1}+\delta_{1 l}-\delta_{1 k}, \cdots, k_{n}+\delta_{n l}-\delta_{n k}\right)$;

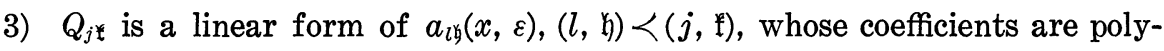
nomials of $p_{l \xi}, h_{1}+\cdots+h_{n}<N$.

Hence $Q_{j \mathfrak{}}$ are known holomorphic functions of $(x, \varepsilon)$ asymptotically developpable in powers of $\varepsilon$.

Let $(j, \mathfrak{l})_{N}$ be the totality of the arrangements $(j, \mathfrak{f})$ such that $1 \leqq j \leqq n$ and $k_{1}+\cdots+k_{n}=N$. We divide $(j, \mathfrak{f})_{N}$ into classes $(j, \mathfrak{f})_{N}{ }^{1}, \cdots,(j, \mathfrak{f})_{N}^{N^{\prime}}$ according to the values of $\lambda_{j \mathfrak{k}}^{\left(\sigma-\sigma \mathfrak{j}^{\prime}\right)}(0)$, i.e. $(j, \mathfrak{f})$ and $\left(j^{\prime}, \mathfrak{f}^{\prime}\right)$ belong to the same class

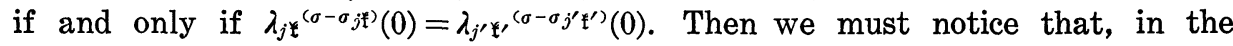
system (4.5), the indices of undetermined $p$ 's appearing on both sides of the equation all belong to the same class. Indeed, such undetermined $p$ 's are divided into following three groups:

(1) $p_{j \mathfrak{k}}$,

(2) $p_{h \mathfrak{\xi}}$ in the term $\varepsilon^{\sigma} \sum a_{j h}(x, \varepsilon) p_{h \mathfrak{\xi}}$,

(3) $p_{j, \mathfrak{f}_{+} \mathfrak{e}_{l}-\mathfrak{e}_{k}}$ in the term $\sum a_{l k}(x, \varepsilon) p_{j, \mathfrak{x}_{+} \mathfrak{e}_{l}-\mathfrak{e}_{k}}$.

To the group (2) belong $p_{h \mathfrak{x}}$ 's such that $a_{j h}(x, \varepsilon) \neq 0$. Since, according to Theorem 2, we may suppose that linear parts of (4.5) are in a reduced form, i.e. $a_{j h}(x, \varepsilon) \neq 0$ implies $\sigma_{j h}>0$ and $\lambda_{j h}{ }^{\left(\sigma-\sigma \sigma^{h}\right)}(0)=0$, we have

$$
\lambda_{j \mathfrak{\xi}}^{\left(\sigma-\sigma_{j \mathfrak{}}\right)}(0)-\lambda_{h \mathfrak{\xi}}{ }^{\left(\sigma-\sigma_{j \mathfrak{f}}\right)}(0)=\lambda_{j h}{ }^{\left(\sigma-\sigma_{j h}\right)}(0)=0 .
$$

Therefore, every $(h, \mathfrak{l})$ in the group (2) belong to the same class as $(j, \mathfrak{l})$.

To compare the index $\left(j, \mathfrak{l}+\mathfrak{e}_{l}-\mathfrak{e}_{k}\right)$ and $(j, \mathfrak{l})$ in the group (3), we notice that

Hence

$$
\lambda_{\jmath} \mathfrak{x}_{+\mathfrak{e}_{l}-\mathfrak{e}_{k}}-\lambda_{k}=\lambda_{k}-\lambda_{l}=\lambda_{k l}
$$

$$
\lambda_{j, \mathfrak{f}+\mathfrak{l}_{l}-\mathfrak{e}_{k}}^{\left(\sigma-\mathfrak{e}_{k}\right)}(0)-\lambda_{j \mathfrak{t}}^{\left(\sigma-\sigma_{j \mathfrak{l}}\right)}(0)=\lambda_{k l}{ }^{\left(\sigma-\sigma_{k l}\right)}(0) .
$$

As $a_{l k}(x, \varepsilon) \neq 0$ implies $\lambda_{k l}{ }^{(\sigma-\sigma k l)}(0)=0$ as before, $\left(j, \mathfrak{f}+\mathfrak{e}_{l}-\mathfrak{e}_{k}\right)$ and $(j, \mathfrak{l})$ belong to the same class.

Now our purpose is to determine the functions $p_{j \mathfrak{K}}$ from (4.5) under the assumption that

$$
c_{j \mathfrak{k}}(x, \varepsilon) \equiv 0 \quad \text { if } \quad \sigma_{j \mathfrak{k}}=0 \quad \text { or } \quad \lambda_{j \mathfrak{k}}^{\left(\sigma-\sigma_{j \mathfrak{f}}\right)}(0) \neq 0 .
$$


As a first step, we put

$$
p_{l \zeta}(x, \varepsilon) \equiv 0 \quad \text { if } \quad \sigma_{l \zeta}>0 \text { and } \lambda_{l \mathscr{\zeta}}{ }^{\left(\sigma-\sigma_{l \zeta}\right)}(0)=0,
$$

and consider the equation (4.5) in which the index $(j, \mathfrak{l})$ is such that

$$
\lambda_{j \mathfrak{}}(x, \varepsilon) \equiv 0, \quad \text { i.e. } \quad \sigma_{j \mathfrak{k}}=0, \quad \lambda_{j \mathfrak{k}}^{\left(\sigma-\sigma_{j \mathfrak{f}}\right)}(0)=0 .
$$

Then, as we have remarked above, the indices of undetermined $p$ 's in this equation all belong to the same class. Therefore, every undetermined $p_{l \xi}$ in this equation, we have

$$
\lambda_{l \mathfrak{b}}\left(\sigma-\sigma_{l \mathfrak{G})}(0)=0 .\right.
$$

However, on account of the relation (4.7), such $p_{l y}$ for which $\sigma_{l y}>0$ are all equal to zero. Hence, on both sides of the equation (4.5), only the $p_{l y}$ such that $\lambda_{l g}(x, \varepsilon) \equiv 0$ are present. Therefore the totality of such equations forms a self-contained system, and the $p_{j \mathfrak{k}}$ such that

$$
\lambda_{j \mathfrak{H}}(x, \varepsilon) \equiv 0
$$

can be determined by solving this system. Since (4.8) holds for this system, we see (by dividing both sides of the equation by $\varepsilon^{\sigma}$ ) that such $p_{j \mathfrak{k}}$ are holomorphic functions of $(x, \varepsilon)$ in the domain $(4.2)_{\mathfrak{x}}$ where $\boldsymbol{r}_{\mathfrak{x}}, \theta_{-\mathfrak{x}}, \theta_{+\mathfrak{x}}, \delta_{\mathfrak{z}}$ are to be suitably chosen. Obviously they are asymptotically developpable in a form

$$
p_{j \mathfrak{k}} \approx \sum_{p=0}^{\infty} p_{j \mathfrak{k}}(p)(x) \varepsilon^{p} .
$$

Next we consider the index $(j, \mathfrak{l})$ for which

$$
\sigma_{j \mathfrak{t}}>0 \text { and } \lambda_{j \mathfrak{k}}^{\left(\sigma-\sigma_{j \mathfrak{F}}\right)}(0)=0 \text {. }
$$

Since, in this case, we have put $p_{j \mathfrak{x}} \equiv 0$, every $p_{l \mathfrak{l}}$ actually remaining in the equation has the index $(l, \mathfrak{h})$ for which

$$
\sigma_{l \xi}=0 \text {, i.e. } \lambda_{l \xi}(x, \varepsilon) \equiv 0 \text {. }
$$

As such $p_{l \zeta}$ have been all determined in our first step, (4.5) is only an algebraic equation from which we determine $c_{j \mathfrak{x}}(x, \varepsilon)$ such that

$$
\sigma_{j \mathfrak{U}}>0 \text { and } \lambda_{j \mathfrak{k}}^{\left(\sigma-\sigma_{j \mathfrak{f}}\right)}(0)=0 \text {. }
$$

Combining this with (4.6), all $c_{j \mathfrak{}}(x, \varepsilon)$ have been now determined.

Finally, we have to consider the index $(j, \mathfrak{l})$ for which

$$
\sigma_{j \mathfrak{l}}>0 \text { and } \lambda_{j \mathfrak{f}}^{\left(\sigma-\sigma_{j \mathfrak{f}}\right)}(0) \neq 0 .
$$

Since, in this case, $c_{j \mathfrak{k}} \equiv 0$ and $\lambda_{j \mathfrak{k}}^{\left(\sigma-\sigma_{j \mathfrak{}}\right)}(0) \neq 0$, the equation (4.5) is of the same form with (3.7). Therefore, following the same reasoning as in section 3 , we can prove the existence of the formal solution

$$
p_{j \mathfrak{k}} \approx \sum_{p=\sigma j \mathfrak{k}}^{\infty} p_{j \mathfrak{k}}{ }^{(p)}(x) \varepsilon^{p} .
$$

Then, by Theorem 1, there exists a solution $p_{j \mathfrak{k}}(x, \varepsilon)$ holomorphic in $(x, \varepsilon)$ in the domain (4.2) $\mathfrak{x}$ and asymptotically developpable in the form (4.9). Thus we 
have determined all $p_{j \mathfrak{x}}$ and the proof is complete.

Proof of the Corollary. Let us put

$$
\begin{gathered}
y_{n+1}=1, \quad \lambda_{n+1}(x, \varepsilon) \equiv 0, \quad f_{n+1}(x, y, \varepsilon) \equiv 0 ; \\
a_{n+1, k}(x, \varepsilon) \equiv 0 ; \quad 1 \leqq k \leqq n+1, \\
a_{\jmath, n+1}(x, \varepsilon) \equiv a_{j}(x, \varepsilon) ; \quad 1 \leqq j \leqq n .
\end{gathered}
$$

The equation (2.1) can be rewritten as follows:

$$
\varepsilon^{\sigma} \frac{d y_{j}}{d x}=\lambda_{\jmath}(x, \varepsilon) y_{\jmath}+\varepsilon^{\sigma} \sum_{k=1}^{n+1} a_{j k}(x, \varepsilon) y_{k}+\varepsilon^{\sigma} f_{j}\left(x, y_{1}, \cdots, y_{n}, \varepsilon\right), \quad j=1, \cdots, n+1
$$

Without loss of generality, we may assume

$$
\begin{array}{ll}
\lambda_{j}(x, \varepsilon) \neq 0, & j=1, \cdots, n^{\prime}, \\
\lambda_{j}(x, \varepsilon) \equiv 0, & j=n^{\prime}+1, \cdots, n+1 .
\end{array}
$$

By Theorem 2, a linear substitution with holomorphic coefficients

$$
y_{\jmath}=z_{\jmath}+\sum_{k=1}^{n+1} p_{j k}(x, \varepsilon) z_{k}, \quad j=1, \cdots, n+1,
$$

can be so determined that (4.10) is reduced to

$$
\varepsilon^{\sigma} \frac{d z_{J}}{d x}=\lambda_{j}(x, \varepsilon) z_{\jmath}+\varepsilon^{\sigma} \sum_{\lambda_{j k 0}=0, \sigma j k>0} b_{j k}(x, \varepsilon) z_{k}+\varepsilon^{\sigma} g_{j}\left(x, z_{1}, \cdots, z_{n+1} \varepsilon\right)
$$

where $g_{j}\left(x, z_{1}, \cdots, z_{n+1}, \varepsilon\right)$ are holomorphic functions satisfying the same condition as $f_{j}\left(x, y_{1}, \cdots, y_{n}, \varepsilon\right)$.

Owing to the Theorem 3 just proved, we see that the system (4.12) can be formally reduced to the system

$$
\varepsilon^{\sigma} \frac{d u_{\jmath}}{d x} \approx \lambda_{j}(x, \varepsilon) u_{\jmath}+\varepsilon^{\sigma} \sum b_{j k}(x, \varepsilon)+\varepsilon^{\sigma} \sum c_{j k_{1} \cdots k_{n+1}}(x, \varepsilon) u_{1}^{k_{1}} \cdots u_{n+1}^{k_{n+1}}
$$

by a formal transformation of the type

$$
z_{\jmath} \approx u_{\jmath}+\sum p_{j \mathfrak{k}}(x, \varepsilon) u_{1}^{k_{1}} \cdots u_{n+1}^{k_{n+1}} .
$$

Here $c_{j k_{1} \cdots k_{n+1}}(x, \varepsilon) \neq 0$ implies $\sigma_{j \mathfrak{k}}>0$ and $\lambda_{j \mathfrak{k}}^{\left(\sigma-\sigma_{j \mathfrak{k}}\right)}(0)=0$. Thus the first $n^{\prime}$ equations of (4.13) are satisfied by putting

$$
u_{1}=\cdots=u_{n^{\prime}}=0 \text {. }
$$

Then, since $b_{j k}(x, \varepsilon) \equiv 0$ for $j=n^{\prime}+1, \cdots, n+1$, remaining equations are written as

$$
\frac{d u_{J}}{d x}=\sum_{k_{n^{\prime}+1^{+}} \cdots+k_{n+1} \geqq 2} c_{j 0 \cdots k_{n^{\prime}+1} \cdots k_{n+1}}(x, \varepsilon) u_{n^{\prime}+1}^{k_{n^{\prime}+1}} \cdots u_{n+1}^{k_{n+1}} .
$$

Applying Theorem 3, if necessary, to this system, all of the coefficients of the transformed equations can be reduced to zero. Hence, without loss of generality, we may assume that

$$
c_{j 0 \cdots 0 k_{n^{\prime}+1} \cdots k_{n+1}} \equiv 0 \quad \text { for } \quad j=n^{\prime}+1, \cdots, n+1,
$$


in (4.13).

Solving $z_{n+1}$ from the last relation of (4.11) (where we put $y_{n+1}=1$ ) and substituting it into the remaining equations, we have

$$
y_{\jmath}=z_{\jmath}+\sum_{k=1}^{n} Q_{j k}(x, \varepsilon) z_{k}, \quad \jmath=1, \cdots, n .
$$

Therefore, substituting (4.14) into this, we have

Since

$$
y_{j} \approx u_{j}+\sum R_{j \mathfrak{k}}(x, \varepsilon) u_{1}^{k_{1}} \cdots u_{n+1}^{k_{n+1}} .
$$

is evidently a solution of a formally transformed system (4.13), we obtain a formal solution of (4.10) substituting (4.16) into (4.15) which can be written in the form

$$
y_{j} \approx \sum p_{j}{ }^{(k)}(x) \varepsilon^{k}, \quad j=1, \cdots, n .
$$

Thus we have the required result.

5. Investigation of the reduced system. In this section, we shall prove several lemmas concerning the reduced system (4.3) which will be of great avail in proving our main theorem.

In section 2, we have supposed that

$$
\begin{cases}\sigma_{\jmath}=0, & j=n^{\prime}+1, \cdots, n^{\prime \prime}-1, \\ \sigma_{\jmath}>0, & \text { otherwise. }\end{cases}
$$

Here we add further assumptions:

$$
\begin{aligned}
& \sigma_{1}=\cdots=\sigma_{\alpha_{1}}=\sigma_{1}{ }^{*}, \sigma_{\alpha_{1}+1}=\cdots=\sigma_{\alpha_{2}}=\sigma_{2}{ }^{*}, \cdots, \quad \sigma_{\alpha_{h-1+1}}=\cdots=\sigma_{\alpha_{i}}=\sigma_{h}{ }^{*}, \\
& \alpha_{h}=n^{\prime}, \quad \sigma_{1}{ }^{*}>\sigma_{2}{ }^{*}>\cdots>\sigma_{h}{ }^{*}>0 \\
& \sigma_{\alpha_{h+1}}=\cdots=\sigma_{\alpha_{h+1}}=\sigma_{h+1}^{*} \\
& \alpha_{h_{+1}}=n^{\prime \prime}-1, \quad \sigma_{h+1}^{*}=0 \\
& \sigma_{\alpha_{h+1+1}}=\cdots=\sigma_{\alpha_{h+2}}=\sigma_{h+2}^{*}, \cdots, \quad \sigma_{\alpha_{m-1+1}}=\cdots=\sigma_{\alpha_{m}}=\sigma_{m}^{*}, \\
& \alpha_{m}=\sigma, \quad 0<\sigma_{h+2}^{*}<\sigma_{h+3}^{*}<\cdots<\sigma_{m}^{*} .
\end{aligned}
$$

This is clearly consistent with the assumption (2.7) we have hitherto used if $|\varepsilon|$ is chosen sufficiently small.

For convenience' sake, we adopt a notation $(4.3)_{j}$ to signify the $j$-th equation of the system (4.3).

Now let us begin with the proof of the following

LEMMA 2. If we put

$$
u_{k}=0, \quad k \neq \alpha_{\nu-1}+1, \cdots, \alpha_{\nu},
$$

then the equations $(4.3)_{j}\left(j \neq \alpha_{\nu-1}+1, \cdots, \alpha_{\nu}\right)$ are satisfied.

Proof. First consider the case $\alpha_{\nu}<n^{\prime \prime}$. Since the linear part of the 
system (4.3) is already in the reduced from, $a_{j k}(x, \varepsilon) \neq 0$ implies

$$
\sigma_{j k}>0 \text { and } \lambda_{j k}{ }^{\left(\sigma-\sigma_{j k}\right)}(0)=0,
$$

or, what is the same thing,

$$
\sigma_{j}=\sigma_{k}, \quad \lambda_{\jmath}^{\left(\sigma-\sigma_{j}\right)}(0)=\lambda_{k}^{\left(\sigma-\sigma_{k}\right)}(0) .
$$

Therefore, if $a_{j k}(x, \varepsilon) \neq 0$ for $j \neq \alpha_{\nu-1}+1, \cdots, \alpha_{\nu}$, we must have $k \neq \alpha_{\nu-1}+1$, $\cdots, \alpha_{\nu}$, because, according to (5.1), we have

$$
\sigma_{k}=\sigma_{\nu} * \sigma_{\jmath}\left(j \neq \alpha_{\nu-1}+1, \cdots, \alpha_{\nu}\right) \quad \text { if } \quad \alpha_{\nu-1}+1 \leqq k \leqq \alpha_{\nu} .
$$

So we have $\sum a_{j k}(x, \varepsilon) u_{k} \equiv 0$ if $j \neq \alpha_{\nu-1}+1, \cdots, \alpha_{\nu}$, and $u_{k}=0$ for $k \neq \alpha_{\nu-1}+1$, $\cdots, \alpha_{\nu}$.

Next we shall show that $c_{j \mathfrak{k}}(x, \varepsilon) \equiv c_{j k_{1} \cdots k_{n}}(x, \varepsilon)$ always vanish if $j \neq \alpha_{\nu-1}+1$, $\cdots, \alpha_{\nu}$ and $k_{h}=0$ for $h \neq \alpha_{\nu-1}+1, \cdots, \alpha_{\nu}$.

Indeed, for such index $(j, \mathfrak{l})$,

$$
\lambda_{j \mathfrak{k}}(x, \varepsilon)=\lambda_{j}(x, \varepsilon)-\sum_{\alpha_{\nu}-1<h \leqq \alpha_{\nu}} k_{h} \lambda_{h}(x, \varepsilon) .
$$

If $j \leqq \alpha_{\nu-1}$, we have $\sigma_{\jmath}>\sigma_{h}$ according to (5.1). Therefore

$$
\lambda_{\left.j \mathfrak{t}^{(\sigma-\sigma}{ }^{(j)}\right)}(0)=\lambda_{j}^{\left(\sigma-\sigma_{j}\right)}(0) \neq 0
$$

which shows that $c_{j \mathfrak{k}} \equiv 0$. If $\alpha_{\nu}<j \leqq n^{\prime \prime}-1$, we have $\sigma_{1}<\sigma_{h}$ and $\sigma_{h}=\sigma_{\nu}{ }^{*}$ for $\alpha_{\nu-1}<h \leqq \alpha_{\nu}$. Hence

$$
\lambda_{j \mathfrak{k}}{ }^{\left(\sigma-\sigma_{j \mathfrak{F}}\right)}(0)=-\sum k_{h} \lambda_{h}{ }^{\left(\sigma-\sigma_{h)}\right)}(0)
$$

unless $k_{\alpha_{\nu-1}}=\cdots=k_{\alpha_{\nu}}=0$. Then, from the assumption (2.7), we have

$$
\Re \frac{\lambda_{h}^{\left(\sigma-\sigma_{h}\right)}(0) e^{-\imath \Omega}}{\varepsilon^{\sigma_{\nu}{ }^{*}}} \geqq \tau \quad \text { for } \quad \alpha_{\nu-1}<h \leqq \alpha_{\nu} .
$$

If we notice that $k_{h}$ are all positive integers, the above inequality assures us that

$$
\lambda_{j \mathfrak{\ddagger}}^{\left(\sigma-\sigma_{j \mathfrak{}}\right)}(0)=-\sum k_{h} \lambda_{h}{ }^{\left(\sigma-\sigma_{h}\right)}(0) \neq 0
$$

and hence $c_{j k} \equiv 0$.

If $k_{\alpha_{\nu-1+1}}=\cdots=k_{\alpha_{\nu}}=0$, we have $\sigma_{j \mathfrak{k}}=\sigma_{\jmath}$ and $\lambda_{j \mathfrak{f}}{ }^{\left(\sigma-\sigma_{j \mathfrak{t}}\right)}(0)=\lambda_{\jmath}{ }^{\left(\sigma-\sigma_{j}\right)}(0)$ whence we conclude either $\sigma_{j \mathfrak{k}}=0$ or $\sigma_{j \mathfrak{k}}>0$ and $\lambda_{j \mathfrak{k}}^{\left(\sigma-\sigma_{j \mathfrak{f}}\right)}(0) \neq 0$. In any way, we also have $c_{j \mathfrak{}} \equiv 0$.

Proof of the case $\alpha_{\nu} \geqq n^{\prime \prime}$ will be carried out quite similarly.

Thus the right-hand member of (4.3) $)_{j}$ vanishes identically for $j \neq \alpha_{\nu-1}+1$, $\cdots, \alpha_{\nu}$ and our lemma is proved.

From this lemma, we see that, if we put $u_{k}=0\left(k \neq \alpha_{\nu-1}+1, \cdots, \alpha_{\nu}\right)$, the system (4.3) will be reduced to

$$
\begin{array}{r}
\varepsilon^{\sigma} \frac{d u_{j}}{d x}=\lambda_{j}(x, \varepsilon) u_{\jmath}+\varepsilon^{\sigma} \sum a_{j k}(x, \varepsilon) u_{k}+\varepsilon^{\sigma} \sum c_{j \mathfrak{Y}_{\nu}}(x, \varepsilon) u_{\alpha^{\prime}}{ }^{k}{ }_{\alpha^{\prime}} \cdots u_{\alpha^{\prime \prime}}{ }^{k} \alpha_{\alpha^{\prime \prime}}, \\
j=\alpha_{\nu-1}+1, \cdots, \alpha_{\nu},
\end{array}
$$

or, especially when $j=n^{\prime}+1, \cdots, n^{\prime \prime}-1$, to 


$$
\varepsilon^{\sigma} \frac{d u_{\jmath}}{d x}=0,
$$

where we used the following notation for simplicity's sake:

$$
\left(\alpha^{\prime}, \cdots, \alpha^{\prime \prime}\right)=\left(\alpha_{\nu-1}+1, \cdots, \alpha_{\nu}\right), \quad \mathfrak{l}_{\nu}=\left(0, \cdots, 0, k_{\alpha^{\prime}}, \cdots, k_{\alpha^{\prime \prime}}, 0, \cdots, 0\right) .
$$

In order that $c_{j \mathfrak{x}_{\nu}}(x, \varepsilon)$ should not vanish, we must have

$$
\lambda_{j \Psi_{\nu}}{ }^{\left(\sigma-\sigma_{j} \dot{t}_{\nu}\right)}(0) \equiv \lambda_{j}{ }^{\left(\sigma-\sigma_{j}\right)}(0)-\sum k_{h} \lambda_{h}{ }^{\left(\sigma-\sigma_{h}\right)}(0)=0 .
$$

According to the assumption (2.7), such arrangements $\left(0, \cdots, 0, k_{\alpha^{\prime}}, \cdots, k_{\alpha^{\prime \prime}}, 0\right.$, $\cdots, 0)$ are only finite in number, whence we have

LEMMA 3. The left-hand members of the system (5.2), are polynomials in $u_{\alpha^{\prime}}, \cdots, u_{\alpha^{\prime \prime}}$ whose coefficients are functions of $(x, \varepsilon)$ holomorphic in the domain $(4.2)_{\mathfrak{t}_{\nu}}$,

Moreover, we can prove

Lemma 4. The intersection of the domains $(4.2)_{\mathfrak{x}_{\nu}}$ is not empty.

Proof. It suffices to show that the conclusion of the lemma holds for almost all $\left(j, \mathfrak{i}_{\nu}\right)$. First suppose that $\nu \leqq h$.

If $j<\alpha^{\prime}$, we have

$$
\left.\lambda_{j \mathfrak{k}_{\nu}}{ }^{\left(\sigma-\sigma \dot{f}_{\nu}\right)}(0)=\lambda_{j}{ }^{(\sigma-\sigma} \sigma_{j}\right)(0)
$$

Hence, by (2.7), we have

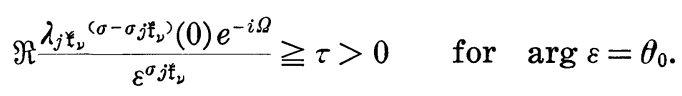

If $\alpha^{\prime} \leqq j \leqq \alpha^{\prime \prime}$, we have

$$
\lambda_{j \mathfrak{t}_{\nu}}{ }^{(\sigma-\sigma}{ }_{\left.j \mathfrak{j}_{\nu}\right)}(0)=\lambda_{j}{ }^{(\sigma-\sigma j)}-k_{\alpha^{\prime}} \lambda_{\alpha^{\prime}}{ }^{\left(\sigma-\sigma_{\alpha^{\prime}}\right)}(0)-\cdots-k_{\alpha^{\prime \prime}} \lambda_{\alpha^{\prime \prime}}{ }^{\left(\sigma-\sigma_{\alpha^{\prime \prime}}\right)}(0) .
$$

Therefore, if the integer $N_{\nu}$ is chosen sufficiently large, we have

$$
\Re \frac{\lambda_{j \mathfrak{k}_{\nu}}{ }^{\left(\sigma-\sigma-\sigma \mathfrak{k}_{\nu}{ }^{\prime}(0) e^{-i \Omega}\right.}}{\varepsilon^{\sigma} j \mathfrak{t}_{\nu}}<-\frac{\tau}{2}<0 \quad \text { if } \quad k_{\alpha^{\prime}}+\cdots+k_{\alpha^{\prime \prime}} \geqq N_{\nu} \quad \text { for } \arg \varepsilon=\theta_{0} .
$$

If $\alpha^{\prime \prime}<j \leqq n^{\prime \prime}$,

$$
\lambda_{j \mathfrak{x}_{\nu}}{ }^{(\sigma-\sigma}{ }_{\left.j \mathfrak{j}_{\nu}\right)}(0)=-k_{\alpha^{\prime}} \lambda_{\alpha^{\prime}}{ }^{\left(\sigma-\sigma_{\alpha^{\prime}}\right)}(0)-\cdots-k_{\alpha^{\prime \prime}} \lambda_{\alpha^{\prime \prime}}{ }^{\left(\sigma-\sigma_{\alpha^{\prime \prime}}\right)}(0),
$$

and we have

$$
\Re \frac{\lambda_{j \sharp_{\nu}}{ }^{(\sigma-\sigma}{ }_{j \dot{\ell}_{\nu}{ }^{\prime}}(0) e^{-i \Omega}}{\varepsilon^{\sigma} \dot{\notin}_{\nu}}<-\tau<0 \quad \text { for } \quad \text { arg } \varepsilon=\theta_{0} .
$$

Finally, if $n^{\prime \prime}<j$ we can easily see from (2.7) that

$$
\Re \frac{\lambda_{j \mathfrak{E}_{\nu}}{ }^{(\sigma-\sigma} \sigma_{\left.j \mathfrak{t}_{\nu}\right)}(0) e^{-i \Omega}}{\varepsilon^{\sigma} j \mathfrak{t}_{\nu}}<-\tau<0 \quad \text { for } \arg \varepsilon=\theta_{0} .
$$

Thus we have either 


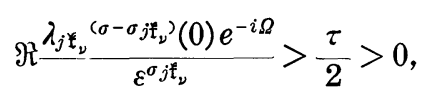

or

$$
\Re \frac{\lambda_{j \mathfrak{k}_{\nu}}{ }^{(\sigma-\sigma} \tilde{j}_{\nu}{ }_{\nu}{ }^{\prime}(0) e^{-i \Omega}}{\varepsilon^{\sigma j \mathfrak{f}_{\nu}}}<-\frac{\tau}{2}<0,
$$

for $\arg \varepsilon=\theta_{0}$, if $k_{\alpha^{\prime}}+\cdots+k_{\alpha^{\prime \prime}} \geqq N_{\nu}$ where $N_{\nu}$ is a sufficiently large positive integer. Therefore, if we construct the donmain (4.2) $)_{\mathfrak{x}_{\nu}}$ by the same method as was used in section 2, the intersection of $\mathfrak{D}\left(\theta_{-\mathfrak{F}_{\nu}}, \theta_{+\mathfrak{F}_{\nu}}, \delta_{\mathfrak{F}_{\nu}}\right),\left(k_{\alpha^{\prime}}+\cdots+k_{\alpha^{\prime \prime}}\right.$ $\geqq N_{\nu}$ ) is not empty and contains the direction arg $\varepsilon=\theta_{0}$. Also the intersection of the domains $|x|<r_{\mathfrak{t}_{\nu}}$ contains a certain neighborhood of the origin.

For $\nu>h+1$, proof is similar, and for $\nu=h+1$, proof is utterly trivial.

From Lemma 4, we have

$$
r_{\nu}{ }^{0}=\inf r_{\mathfrak{t}_{\nu}}>0, \quad \theta_{+\nu}{ }^{0}=\inf \theta_{+\mathfrak{t}_{\nu}}>\theta_{0}>\theta_{-\nu}{ }^{0}=\sup \theta_{-\mathfrak{x}_{\nu}} .
$$

Now consider the lozenge $E(\rho, \varphi, \Omega)$ contained in the domain $|x|<r_{\nu}{ }^{0}$, and define a point $x_{\nu}{ }^{*}$ by

$$
x_{\nu}^{*}= \begin{cases}a^{(3)} & (\nu<h+1) \\ a^{(1)} & (\nu>h+1) .\end{cases}
$$

Let $\Gamma_{x_{0}}{ }^{(\nu)}$ be the segment joining $x_{0}$ and $x_{\nu}{ }^{*}$. Then the variable point on this segment is contained in the domain $E(\rho, \varphi, \Omega)$ and is expressed as

$$
x=x_{\nu}{ }^{*}+s e^{2 \psi}
$$

where $s$ is the length of the segment from $x_{\nu}{ }^{*}$ to $x$, and $\psi=\arg \left(x_{0}-x_{\nu}{ }^{*}\right)$.

Let

$$
u_{\jmath}=U_{j}\left(x, x_{0} u_{\jmath}^{0}, \varepsilon\right), \quad j=\alpha^{\prime}, \cdots, \alpha^{\prime \prime},
$$

be the holomorphic solution of the system $(5.2)_{\nu}$ such that

$$
u_{\jmath}=u_{\jmath}{ }^{0} \quad \text { for } \quad x=x_{0},
$$

$\left(x_{0}, \varepsilon\right)$ being an arbitrary point in the domain

$$
E(\rho, \varphi, \Omega) \times \mathfrak{D}\left(\theta_{-\nu}{ }^{0}, \theta_{+\nu}{ }^{0}, \delta_{\nu}{ }^{0}\right) .
$$

If, especially, $\left(\alpha^{\prime}, \cdots, \alpha^{\prime \prime}\right)=\left(n^{\prime}+1, \cdots, n^{\prime \prime}-1\right), u_{\jmath}$ do not depend on $x$.

Then, from Theorem 3 and the lemmas just proved above, we have

Lemma 5. There exist $m$ systems of formal solutions $\left(\mathrm{F}_{\nu}\right), \nu=1, \cdots, m$, of $(1.1)$

$\left(\mathrm{F}_{\nu}\right)$

$$
y_{\jmath} \approx \delta_{j}{ }^{(\nu)} u_{j}+\sum p_{j \varkappa_{\nu}}(x, \varepsilon) u_{\alpha^{\prime}}{ }^{k_{\alpha^{\prime}}} \cdots u_{\alpha^{\prime \prime}}{ }^{k_{\alpha^{\prime \prime}},}, \quad j=1, \cdots, n
$$

where

$$
\delta_{j}^{(\nu)}= \begin{cases}1, & j=\alpha^{\prime}, \cdots, \alpha^{\prime \prime}, \\ 0, & \text { otherwise, }\end{cases}
$$

and the coefficients $p_{j \ddagger_{\nu}}(x, \varepsilon)$ are functions of $(x, \varepsilon)$ holomorphic in the domain 


$$
|x|<r_{\nu}{ }^{0}, \quad \varepsilon \in \mathfrak{D}\left(\theta_{-\nu}{ }^{0}, \theta_{+\nu}{ }^{0}, \delta_{\nu}{ }^{0}\right),
$$

and asymptotically developpable in the form

$$
p_{j \mathfrak{k}_{\nu}}(x, \varepsilon) \approx \sum_{p=\sigma_{j ; \mathfrak{j}_{\nu}}}^{\infty} p_{j \mathfrak{k}_{\nu}}{ }^{(p)}(x) \varepsilon^{p}
$$

as $\varepsilon \rightarrow 0$ in the domain $(5.6)_{\nu}$.

Now we shall prove our final lemma.

LEMMA 6. If $\sigma_{\nu}{ }^{*}>0$, the inequality

$$
\frac{d}{d s}\left\{\max _{k=\alpha^{\prime}}^{\alpha^{\prime \prime}}\left|u_{k}\right|^{1 / \mu_{i}}\right\} \geqq \frac{\sin \gamma}{4|\varepsilon|^{\sigma_{\nu}^{*}}} \min _{k=\alpha^{\prime}}^{\alpha^{\prime \prime}} \frac{\left|\lambda_{k}{ }^{\left(\sigma-\sigma_{k}\right)}(0)\right|}{\mu_{k}} \max _{k=\alpha^{\prime}}^{\alpha^{\prime \prime}}\left|u_{k}\right|^{1 / \mu_{k}}
$$

holds for $x$ on $\Gamma_{x_{0}}{ }^{(\nu)}$ and $\varepsilon \in \mathfrak{D}\left(\theta_{-\nu}{ }^{0},{\theta_{+\nu}}{ }^{0}, \delta_{\nu}{ }^{0}\right)$ where $r$ is a small positive number determined in the same way as in section 2 , and

$$
\mu_{k}=\left\{\begin{aligned}
\Re \frac{\lambda_{k}{ }^{\left(\sigma-\sigma_{k}\right)}(0) e^{-i \Omega}}{\left(\delta_{0} e^{\left.i \theta_{0}\right)^{\sigma}}\right.}, & k<\alpha_{h+1}, \\
1, & k=\alpha_{h+1}, \\
-\Re \frac{\lambda_{k}{ }^{\left(\sigma-\sigma_{k}\right)}(0) e^{-i \Omega}}{\left(\delta_{0} e^{\left.i \theta_{0}\right)^{\sigma}}\right.}, & k>\alpha_{h+1},
\end{aligned}\right.
$$

$\delta_{0}$ being a sufficiently small positive number.

Proof. We give the proof for the case $\nu>h+1$ only. Other cases will be treated analogously.

Since the $c_{j \varkappa_{\nu}}(x, \varepsilon)$ appearing in the right-hand member of $(5.2)_{\nu}$ are finite in number, the inequality

$$
\sum\left|a_{j k}(x, \varepsilon)\right|+\sum\left|c_{j \mathfrak{t}_{\nu}}(x, \varepsilon)\right|<\frac{\left|\lambda_{j}{ }^{\left(\sigma-\sigma_{j}\right)}(0)\right| \sin \gamma}{4|\varepsilon|^{\sigma_{\nu}^{*}}}
$$

will hold in the domain $(5.6)_{\nu}$ if $\delta_{\nu}{ }^{0}$ is chosen sufficiently small.

Moreover, we have

$$
\begin{aligned}
& \Re \frac{\lambda_{j}(x, \varepsilon) e^{\imath \psi}}{\varepsilon^{\sigma_{\nu}{ }^{*}}}=\Re \frac{\lambda_{\jmath}^{\left(\sigma-\sigma_{j}\right)}(0) e^{\imath \psi}}{\varepsilon^{\sigma_{\nu}{ }^{*}}}+O\left(\frac{x}{\varepsilon^{\sigma_{\nu}^{*}}}\right), \\
& -\varphi \leqq \varphi+\Omega \leqq \varphi, \\
& \varphi-\frac{\pi}{2}+\gamma \leqq \arg \lambda_{\jmath}{ }^{\left(\sigma-\sigma_{j}\right)}(0)-\Omega-\sigma_{\nu}{ }^{*} \arg \varepsilon \leqq-\varphi+\frac{\pi}{2}-\gamma, \quad j=\alpha^{\prime}, \cdots, \alpha^{\prime \prime},
\end{aligned}
$$

in the domain (5.6) $)_{\nu}$. Hence we have

$$
\left|\arg \frac{\lambda_{\jmath}{ }^{\left(\sigma-\sigma_{j}\right)}(0) e^{\imath \psi}}{\varepsilon^{\sigma_{\nu}^{*}}}\right| \leqq \frac{\pi}{2}-\gamma
$$

which implies

$$
\cos \left(\arg \left(\frac{\lambda_{j}^{\left(\sigma-\sigma_{j}\right)}(0) e^{\imath \psi}}{\varepsilon^{\sigma_{\nu}^{*}}}\right)\right) \geqq \sin \gamma>0 .
$$

Accordingly, if $\delta_{\nu}{ }^{0}$ and $r_{\nu}{ }^{0}$ are sufficiently small, 


$$
\Re \frac{\lambda_{j}(x, \varepsilon) e^{\imath \psi} \psi}{\varepsilon^{\sigma_{\nu}{ }^{*}}} \geqq \frac{\left|\lambda_{\jmath}{ }^{\left(\sigma-\sigma_{j}^{*}\right)}(0)\right| \sin \gamma}{2|\varepsilon|^{\sigma_{\nu}^{*}}} .
$$

If we choose a point $x^{0}$ arbitrarily on $\Gamma_{x_{0}}{ }^{(\nu)}$, there exists an index $k_{0}$ such that

$$
\max _{k=\alpha^{\prime}}^{\alpha^{\prime \prime}}\left|u_{k}\right|^{1 / \mu_{k}}=\left|u_{k_{0}}\right|^{1 / \mu_{\kappa_{0}}}
$$

Naturally $k_{0}$ depends upon the choicee of $x^{0}$. The conditions

respectively imply

$$
c_{k_{0} \sharp_{\nu}}(x, \varepsilon) \neq 0 \quad \text { and } \quad a_{k_{0} k}(x, \varepsilon) \neq 0
$$

$$
\left|u_{\alpha^{\prime}}\right|^{k_{\alpha^{\prime}}} \cdots \cdots\left|u_{\alpha^{\prime \prime}}\right|^{k_{\alpha^{\prime \prime}}} \leqq\left|u_{k_{0}}\right| \text { and }\left|u_{k}\right| \leqq\left|u_{k_{0}}\right|
$$

at the point $x^{0}$. Indeed, suppose that $c_{k_{0} \mathfrak{F}_{\nu}}(x, \varepsilon) \neq 0$. Then, as we already know, we must have

or, from (5.9),

$$
\lambda_{k_{0}}{ }^{\left(\sigma-\sigma_{k_{0}}\right)}(0)=k_{\alpha^{\prime}} \lambda_{\alpha^{\prime}}{ }^{\left(\sigma-\sigma_{\alpha^{\prime}}\right)}(0)+\cdots+k_{\alpha^{\prime \prime}} \lambda_{\alpha^{\prime \prime}}{ }^{\left(\sigma-\sigma_{\alpha^{\prime \prime}}\right)}(0),
$$

Thus we obtain

$$
\mu_{k_{0}}=k_{\alpha^{\prime}} \mu_{\alpha^{\prime}}+\cdots+k_{\alpha^{\prime}} \mu_{\alpha^{\prime \prime}}
$$

$$
\begin{aligned}
\left|u_{k_{0}}\right| & =\left(\left|u_{k_{0}}\right|^{1 / \mu_{k_{0}}}\right)^{k_{\alpha^{\prime}} \mu_{\alpha^{\prime}}} \cdots\left(\left|u_{k_{0}}\right|^{1 / \mu_{k_{0}}}\right)^{k_{\alpha^{\prime}} \mu_{\alpha^{\prime \prime}}} \\
& \geqq\left(\left|u_{\alpha^{\prime}}\right|^{1 / \mu_{\alpha^{\prime}}}\right)^{k_{\alpha^{\prime}} \mu_{\alpha^{\prime}}} \cdots\left(\left|u_{\alpha^{\prime \prime}}\right|^{1 / \mu_{\alpha^{\prime \prime}}}\right)^{k_{\alpha^{\prime}} \mu_{\alpha^{\prime \prime}}} \\
& =\left.\left|u_{\alpha^{\prime}}\right|^{k_{\alpha^{\prime}} \cdots \mid u_{\alpha^{\prime \prime}}}\right|^{k_{\alpha^{\prime \prime}}},
\end{aligned}
$$

which is the first inequality of (5.12).

The second inequality of (5.12) can be proved similarly.

Now the inequality (5.8) can be proved easily. Let $s^{0}$ be the length of the segment $\overline{x_{\nu}{ }^{*} x^{0}}$. Then

$$
\begin{aligned}
& {\left[\frac{d}{d s}\left\{\max \left|u_{k}\right|^{1 / \mu_{k}}\right\}\right]_{s=s^{0}}=\left[\frac{d}{d s}\left|u_{k_{0}}\right|^{1 \mu_{k_{0}}}\right]_{s=s^{0}} } \\
= & {\left[\frac{1}{\mu_{k_{0}}}\left|u_{k_{0}}\right|^{1 / \mu_{k 0} 0} \frac{1}{\left|u_{k_{0}}\right|} \frac{d}{d s}\left|u_{k_{0}}\right|\right]_{s=s^{0}}=\frac{1}{\mu_{k_{0}}}\left[\max \left|u_{k}\right|^{1 / \mu_{k}} \Re\left(\frac{d}{d s} \log u_{k_{0}}\right)\right]_{s=s^{0}} } \\
= & \frac{1}{\mu_{k_{0}}}\left[\max \left|u_{k}\right|^{1 / \mu_{k}} \Re\left(\frac{1}{u_{k_{0}}} \frac{d u_{k_{0}}}{d x} e^{\imath \psi}\right)\right]_{x=x 0} .
\end{aligned}
$$

Making use of the equation (5.2), we have

Thus, from (5.10), (5.11) and (5.12),

$$
\begin{aligned}
\Re\left(\frac{1}{u_{k_{0}}} \frac{d u_{k_{0}}}{d x} e^{\imath \psi}\right) \geqq & \Re\left(\frac{\lambda_{k_{0}}(x, \varepsilon)}{\varepsilon^{\sigma}} e^{\imath \psi}\right)-\left\{\sum\left|a_{k_{0} k}(x, \varepsilon)\right| \frac{\left|u_{k}\right|}{\left|u_{k_{0}}\right|}\right. \\
& \left.+\sum\left|c_{k_{0} \mathfrak{f}_{\nu}}(x, \varepsilon)\right| \frac{\left|u_{\alpha^{\prime}} k_{\alpha^{\prime}} \cdots u_{\alpha^{\prime \prime}} k_{\alpha^{\prime \prime}}\right|}{\left|u_{k_{0}}\right|}\right\} .
\end{aligned}
$$

$$
\Re\left(\frac{1}{u_{k_{0}}} \frac{d u_{k_{0}}}{d x} e^{\imath \psi}\right) \geqq \frac{\left|\lambda_{k_{0}}{ }^{(\sigma-\sigma}{ }_{k_{0}}{ }^{\prime}(0)\right| \sin \gamma}{2|\varepsilon|^{\sigma_{\nu}{ }^{*}}}-\frac{\left|\lambda_{k}{ }^{\left(\sigma-\sigma_{k_{0}}\right)}(0)\right| \sin \gamma}{4|\varepsilon|_{\nu}^{\sigma_{\nu}{ }^{*}}}=\frac{\left|\lambda_{k_{0}}{ }^{\left(\sigma-\sigma_{k_{0}}\right.}(0)\right| \sin \gamma}{4|\varepsilon|^{\sigma_{\nu}{ }^{*}}}
$$


whence immediately follows the required inequality.

6. Main theorem. Now we are in a position to undertake the proof of our main theorem which will by stated as follows:

THEOREM 4. In the expressions of the formal solutions $\left(\mathrm{F}_{1}\right),\left(\mathrm{F}_{h+1}\right)$ and $\left(\mathrm{F}_{m}\right)$, the formal power series in their right-hand members converge uniformly in the domain

$$
|x|<r_{0}, \quad \max \left|u_{k}\right|^{1 / \mu_{k}}<\zeta_{0}, \quad \varepsilon \in \mathfrak{D}\left(\theta_{0}-a, \theta_{0}+a, \delta_{0}\right)
$$

where $r_{0}, \zeta_{0}, a$ and $\delta_{0}$ are sufficiently small positive constants.

In other words, formal solutions $\left(\mathrm{F}_{1}\right),\left(\mathrm{F}_{h+1}\right)$ and $\left(\mathrm{F}_{m}\right)$ represent actual solutions of (1.1).

Proof of this theorem necessitates rather lengthy reasoning and we must proceed step by step.

1) Preliminary transformation. To simplify our description, we make a following convention:

$\left(\beta^{\prime}, \cdots, \beta^{\prime \prime}\right)$ denotes $\left(1, \cdots, \alpha_{1}\right)$ or $\left(n^{\prime}+1, \cdots, n^{\prime \prime}-1\right)$ or $\left(\alpha_{m-1}+1, \cdots, n\right)$, $\mathfrak{f}_{\beta}$ denotes the index $\left(0, \cdots, 0, k_{\beta^{\prime}}, \cdots, k_{\beta^{\prime \prime}}, 0, \cdots, 0\right)$.

Let us put

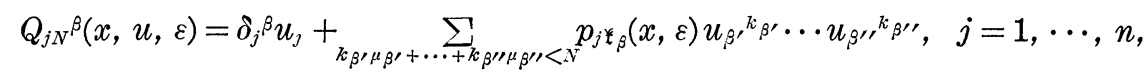

where

$$
\delta_{j}{ }^{\beta}= \begin{cases}0, & j \neq \beta^{\prime}, \cdots, \beta^{\prime \prime}, \\ 1, & \text { otherwise. }\end{cases}
$$

Since $Q_{j N^{\beta}}(x, u, \varepsilon)$ is a polynomial in $u_{\beta^{\prime}}, \cdots, u_{\beta^{\prime \prime}}$ with holomorphic coefficients and $\lim _{\varepsilon \rightarrow 0} Q_{j N^{\beta}} \beta(0,0, \varepsilon)=0$, we can find sufficiently small positive numbers $\gamma_{0}{ }^{\prime}$, $\zeta_{0}{ }^{\prime}, \delta_{0}{ }^{\prime}, \rho_{0}{ }^{\prime}$ and $\eta_{0}{ }^{\prime}$ such that we have

$$
\left|Q_{j N}{ }^{\beta}(x, u, \varepsilon)\right|+\rho_{0}{ }^{\prime}<\eta_{0}{ }^{\prime}\left(\rho_{0}{ }^{\prime}<\eta_{0}{ }^{\prime}\right), \quad j=1, \cdots, n,
$$

in the domain

$$
|x|<r_{0}{ }^{\prime}, \quad \max _{k=\beta^{\prime}}^{\beta^{\prime \prime}}\left|u_{k}\right|^{1 / \mu_{k}}<\zeta_{0}{ }^{\prime}, \quad \varepsilon \in \mathfrak{D}\left(\theta_{-\beta}^{0}, \theta_{+\beta}^{0}, \delta_{0}{ }^{\prime}\right),
$$

where $\mathfrak{D}\left(\theta_{-\beta}^{0}, \theta_{+\beta}^{0}, \delta_{0}^{\prime}\right)$ denotes

$$
\begin{array}{lll}
\mathfrak{D}\left(\theta_{-1}^{0} \theta_{+1}^{0}, \delta_{0}{ }^{\prime}\right) & \text { if } \quad\left(\beta^{\prime}, \cdots, \beta^{\prime \prime}\right)=\left(1, \cdots, \alpha_{1}\right), \\
\mathfrak{D}\left(\theta_{-(h+1)}^{0}, \theta_{+(h+1)}^{0}, \delta_{0}^{\prime}\right) & \text { if } \quad\left(\beta^{\prime}, \cdots, \beta^{\prime \prime}\right)=\left(n^{\prime}+1, \cdots, n^{\prime \prime}-1\right), \\
\mathfrak{D}\left(\theta_{-m}^{0}, \theta_{+m}^{0}, \delta^{\prime}\right) & \text { if } \quad\left(\beta^{\prime}, \cdots, \beta^{\prime \prime}\right)=\left(\alpha_{m-1}+1, \cdots, n\right),
\end{array}
$$

and, moreover, the right-hand members of (1.1) are holomorphic in $(x, y, \varepsilon)$ in the domain

$$
|x|<r_{0}{ }^{\prime}, \quad \max \left|y_{k}\right|<\eta_{0}{ }^{\prime}, \quad \varepsilon \in \mathfrak{D}\left(\theta_{-\beta}^{0}, \theta_{+\beta}^{0}, \delta_{0}{ }^{\prime}\right) .
$$

Let us make a transformation 
(6.3) $y_{j}=Q_{j N^{\beta}}\left(x, U_{\beta^{\prime}}\left(x, x_{0}, u_{\beta^{\prime}}{ }^{0}, \varepsilon\right), \cdots, U_{\beta^{\prime \prime}}\left(x, x_{0}, u_{\beta^{\prime \prime}}, \varepsilon\right), \varepsilon\right)+v_{j}, \quad j=1, \cdots, n$, and let

$$
\varepsilon^{\sigma} \frac{d v_{j}}{d x}=\lambda_{j}(x, \varepsilon) v_{j}+\varepsilon^{\sigma} \sum a_{j k}(x, \varepsilon) v_{k}+\varepsilon^{\sigma} g_{j}\left(x, U_{\beta^{\prime}}, \cdots, U_{\beta^{\prime \prime}}, v_{1}, \cdots, v_{n}, \varepsilon\right)
$$

be the transformed system. Elementary calculation shows that

$$
\begin{aligned}
& \varepsilon^{\sigma} g_{j}(x, u, v, \varepsilon)=\lambda_{j}(x, \varepsilon) Q_{j N} \beta(x, u, \varepsilon)+\varepsilon^{\sigma} \sum a_{j k}(x, \varepsilon) Q_{k N} \beta(x, u, \varepsilon) \\
& +\varepsilon^{\sigma} f_{j}\left(x, Q_{N} \beta(x, u, \varepsilon)+v, \varepsilon\right)-\varepsilon^{\sigma} \frac{\partial Q_{j N^{\beta}}}{\partial x}-\sum_{l=\beta^{\prime}}^{\beta^{\prime \prime}} \frac{\partial Q_{j N^{\beta}}}{\partial u_{l}} \varepsilon^{\sigma} \frac{d u_{l}}{d x} \\
& =G_{1}+G_{2}-G_{3}-G_{4}, \\
& G_{1}=\varepsilon^{\sigma} f_{j}\left(x, Q_{N}^{\beta}+v, \varepsilon\right), \\
& G_{2}=\lambda_{j}(x, \varepsilon)\left\{Q_{j N} \beta(x, u, \varepsilon)-\delta_{j}^{\beta} u_{j}\right\}+\varepsilon^{\sigma} \sum \alpha_{j k}(x, \varepsilon) Q_{k N^{\beta}}{ }^{\beta} \\
& -\sum_{l=\beta^{\prime}}^{\beta^{\prime \prime}} \lambda_{l}(x, \varepsilon) u_{l}\left\{\frac{\partial Q_{j N^{\beta}}}{\partial u_{l}}-\delta_{j l}\right\}-\varepsilon^{\sigma} \frac{\partial Q_{j N^{\beta}}}{\partial x}, \\
& G_{3}=\sum_{l=\beta^{\prime}}^{\beta^{\prime \prime}}\left\{\varepsilon^{\sigma} \frac{d u_{l}}{d x}-\lambda_{l}(x, \varepsilon) u_{l}\right\}\left\{\frac{\partial Q_{j N}{ }^{\beta}}{\partial u_{l}}-\delta_{j l}\right\}, \\
& G_{4}=\sum_{l=\beta^{\prime}}^{\beta^{\prime \prime}} \varepsilon^{\sigma} \frac{d u_{l}}{d x} \delta_{j l}-\lambda_{j}(x, \varepsilon) u_{j} \delta_{j}{ }^{\beta},
\end{aligned}
$$

where $\delta_{j l}$ mean the Kronecker's symbol.

Clearly, $G_{1}, G_{3}$, and $G_{4}$ contain a factor $\varepsilon^{\sigma}$. Moreover, if we notice that $G_{2}$ can be rewritten as

$$
\begin{aligned}
\sum\left[\lambda_{j \mathfrak{k}_{\beta}}(x, \varepsilon) p_{j \mathfrak{x}_{\beta}}(x, \varepsilon)\right. & +\varepsilon^{\sigma} \sum a_{j k}(x, \varepsilon) p_{k \mathfrak{k}_{\beta}}(x, \varepsilon) \\
& \left.-\varepsilon^{\sigma} \frac{d p_{j \mathfrak{x}_{\beta}}(x, \varepsilon)}{d x}\right] u_{\beta^{\prime}}{ }^{{ }^{k}{ }^{\prime}} \cdots u_{\beta^{\prime \prime}}{ }^{k_{\beta^{\prime \prime}}},
\end{aligned}
$$

and that

$$
\begin{aligned}
& \lambda_{j \mathfrak{k}_{\beta}}(x, \varepsilon)=\lambda_{j \mathfrak{j}_{\beta}}{ }^{\left(\sigma-\sigma_{j \mathfrak{f}_{\beta}}\right)}(x) \varepsilon^{\sigma-\sigma_{j \mathfrak{k}}}+O\left(\varepsilon^{\left.\sigma-\sigma_{j \mathfrak{f}_{\beta}+1}\right)},\right. \\
& p_{j \mathfrak{k}_{\beta}}(x, \varepsilon)=p_{j \mathfrak{k}_{\beta}}{ }^{\left(\sigma_{j \mathfrak{k}}\right)}(x) \varepsilon^{\sigma \mathfrak{j}_{\beta}}+O\left(\varepsilon^{\sigma j \mathfrak{f}_{\beta}+1}\right),
\end{aligned}
$$

we can also conclude that $G_{2}$ contains a factor $\varepsilon^{\sigma}$. Hence $g_{j}(x, u, v, \varepsilon)$ are functions of $\left(x, u_{\beta^{\prime}}, \cdots, u_{\beta^{\prime \prime}}, v_{1}, \cdots, v_{n}, \varepsilon\right)$ holomorphic in the domain

$$
|x|<r_{0}{ }^{\prime}, \quad \max _{k=\beta^{\prime}}^{\beta^{\prime \prime}}\left|u_{k}\right|^{1 / \mu_{k}}<\zeta_{0^{\prime}} \max _{j}\left|v_{j}\right|<\rho_{0}^{\prime}, \quad \varepsilon \in \mathfrak{D}\left(\theta_{-\beta}^{0}, \theta_{+\beta}^{0}, \delta_{0}{ }^{\prime}\right) .
$$

On the other hand, the system (6.4) evidently admits a formal solution

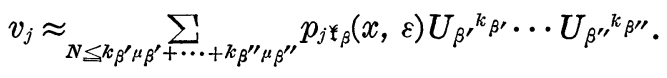

So we can easily see that the positive constants $A_{\jmath}$ and $B_{N}$ can be so chosen that

$$
\left|\sum a_{j k}(x, \varepsilon) v_{k}+g_{j}(x, u, v, \varepsilon)\right|<A_{\jmath} \max _{k}\left|v_{k}\right|+B_{N} \max _{k=\beta^{\prime}}^{\beta^{\prime \prime}}\left|u_{k}\right|^{N / \mu_{k}} .
$$

Next we make a transformation 
where ${ }_{\beta} x^{*}$ is equal to

$$
v_{j}=w_{j} \exp \Lambda_{j}(x, \varepsilon) \quad \Lambda_{j}(x, \varepsilon)=\int_{\beta^{x^{*}}}^{x} \lambda_{j}(x, \varepsilon) \varepsilon^{-\sigma} d x
$$

$$
\begin{aligned}
& x_{1}{ }^{*} \text { when }\left(\beta^{\prime}, \cdots, \beta^{\prime \prime}\right)=\left(1, \cdots, \alpha_{1}\right), \\
& x_{\jmath}{ }^{*} \text { when } \quad\left(\beta^{\prime}, \cdots, \beta^{\prime \prime}\right)=\left(n^{\prime}+1, \cdots, n^{\prime \prime}-1\right), \\
& x_{m}{ }^{*} \text { when }\left(\beta^{\prime}, \cdots, \beta^{\prime \prime}\right)=\left(\alpha_{m-1}+1, \cdots, n\right) .
\end{aligned}
$$

Then the system (6.4) will be reduced to

$$
\begin{aligned}
\frac{d w_{j}}{d x} & =\sum a_{j k}(x, \varepsilon) w_{k} \exp \Lambda_{k}(x, \varepsilon)+g_{j}(x, U, w \exp \Lambda, \varepsilon) \exp \left(-\Lambda_{\jmath}(x, \varepsilon)\right) \\
& =h_{j}(x, U, w \exp \Lambda(x, \varepsilon), \varepsilon) \exp \left(-\Lambda_{j}(x, \varepsilon)\right) .
\end{aligned}
$$

2) Family $\mathfrak{F}$ and the mapping $\mathfrak{T}$. Let us choose the domains $E(\rho, \varphi, \Omega)$ and $\mathfrak{D}\left(\theta_{0}-a, \theta_{0}+a, \delta_{0}\right)$ such that

$$
E(\rho, \varphi, \Omega) \subset\left\{x ;|x|<r_{0}{ }^{\prime}\right\}, \quad \mathfrak{D}\left(\theta_{0}-a, \theta_{0}+a, \delta_{0}\right) \subset \mathfrak{D}\left(\theta_{-\beta}^{0}, \theta_{+\beta}^{0}, \delta_{0}{ }^{\prime}\right) .
$$

Let $₹$ be the family of the systems $\left(\phi_{1}(x, u, \varepsilon), \cdots, \phi_{n}(x, u, \varepsilon)\right)$ where $\phi_{j}(x, u, \varepsilon)$ are functions of $\left(x, u_{\beta^{\prime}} \cdots, u_{\beta^{\prime \prime}}, \varepsilon\right)$ holomorphic in the domain

$$
x \in E(\rho, \varphi, \Omega), \quad \max _{k=\beta^{\prime}}^{\beta^{\prime \prime}}\left|u_{k}\right|^{1 / \mu_{k}}<\zeta_{0}, \quad \varepsilon \in \mathfrak{D}\left(\theta_{0}-a, \theta_{0}+a, \delta_{0}\right)
$$

and satisfying the inequalities

$$
\left|\phi_{j}(x, u, \varepsilon)\right| \leqq K_{N} \max _{k=\beta^{\prime}}\left|u_{k}\right|^{N / \mu_{k}} \exp \left(-\Re \Lambda_{j}(x, \varepsilon)\right), \quad j=1, \cdots, n,
$$

where $0<\zeta_{0}<\zeta_{0}{ }^{\prime}$ and $0<K_{N} \zeta_{0}{ }^{N}<\rho_{0}{ }^{\prime}$. Put

$$
\begin{aligned}
& \bar{\phi}_{J}\left(x_{0}, u^{0}, \varepsilon\right)=\phi_{j}\left({ }_{\beta} x^{*}, U\left({ }_{\beta} x^{*}, x_{0}, u^{0}, \varepsilon\right), \varepsilon\right) \\
& +\int_{\beta^{* *}}^{x_{0}} h_{j}\left(x, U\left(x, x_{0}, u^{0}, \varepsilon\right), \phi\left(x, U\left(x, x_{0}, u^{0}, \varepsilon\right) \exp \Lambda(x, \varepsilon), \varepsilon\right) \exp \left(-\Lambda_{j}(x, \varepsilon)\right) d x,\right.
\end{aligned}
$$

where $\left(x_{0}, u_{\beta^{\prime}}{ }^{0}, \cdots, u_{\beta^{\prime \prime}}{ }^{0}, \varepsilon\right)$ is an arbitrary point in the domain (6.9) and the integration is to be carried out along the segment $\overline{{ }_{\beta} x^{*} x_{0}}$ which will be henceforth denoted by ${ }_{\beta} \Gamma_{x_{0}}$. Then the mapping $\mathfrak{I}$ is defined as follows:

$$
\left(\phi_{1}, \cdots, \phi_{n}\right) \stackrel{\mathfrak{I}}{\rightarrow}\left(\bar{\phi}_{1}, \cdots, \bar{\phi}_{n}\right) \text {. }
$$

Since $\{0, \cdots, 0\} \in \mathfrak{F}, \mathfrak{F}$ is not empty. Further, as can be immediately seen, $\mathfrak{F}$ is convex, closed and normal. On the other hand, since, according to Lemma 6 , $\max _{k=\beta^{\prime}}^{\beta^{\prime \prime}}\left|u_{k}\right|^{1 / \mu_{k}}$ decreases monotonically as $x$ tends to ${ }_{\beta} x^{*}$ on ${ }_{\beta} \Gamma_{x_{0}}$, the integrand

$$
h_{\jmath}(x, U, \phi \exp \Lambda, \varepsilon) \exp \left(-\Lambda_{j}\right)
$$

is bounded. Thus the integral in the right-hand member or (6.11) converges and the mapping $\mathfrak{I}$ has a well-defined meaning.

Our proof of the main theorem is based on the existence of a fixed point of this mapping.

3) Two lemmas. Before going into the essential part of the proof, we must state two lemmas. Suppose that 
$1^{\circ} . \quad D$ and $\mathscr{D}$ are simply connected domains in a complex plane;

$2^{\circ}$. $\mathfrak{D}_{r}$ is a simply connected domain in a product space of $r$ complex planes;

$3^{\circ} . f_{k}\left(x, z_{1}, \cdots, z_{m}, \varepsilon\right), k=1, \cdots, m$, are holomorphic in the domain

$$
x \in D, \quad\left(z_{1}, \cdots, z_{m}\right) \in \mathfrak{D}_{m}, \quad \varepsilon \in \mathfrak{D} ;
$$

$4^{\circ} . g_{k}\left(x, y_{1}, \cdots, y_{n}, \varepsilon\right), k=1, \cdots, n$, are holomorphic in the domain

$$
x \in D, \quad\left(y_{1}, \cdots, y_{n}\right) \in \mathfrak{D}_{n}, \quad \varepsilon \in \mathfrak{D} ;
$$

$5^{\circ} . \phi_{k}\left(x, z_{1}, \cdots, z_{m}, \varepsilon\right), k=1, \cdots, n$, are holomorphic in the domain

$$
x \in D, \quad\left(z_{1} \cdots, z_{m}\right) \in \mathfrak{D}_{m}, \quad \varepsilon \in \mathfrak{D} ;
$$

$6^{\circ} . z_{k}\left(x, x_{0}, z_{1}{ }^{0}, \cdots, z_{m}{ }^{0}, \varepsilon\right), k=1, \cdots, m$, is a solution of the system

$$
\frac{d z_{k}}{d x}=f_{k}\left(x, z_{1} \cdots, z_{m}, \varepsilon\right), \quad k=1, \cdots, m,
$$

such that $z_{k}{ }^{0}=z_{k}\left(x_{0}, x_{0}, z^{0}, \varepsilon\right)$ where $\left(x_{0}, z^{0}, \varepsilon\right) \in D \times \mathfrak{D}_{m} \times \mathfrak{D}$;

$7^{\circ}$. $G_{k}\left(x, x_{0}, z_{1}{ }^{0}, \cdots, z_{m}{ }^{0}, \varepsilon\right) \equiv g_{k}\left(x, \phi_{1}\left(x, z^{0}, \varepsilon\right), \cdots, \phi_{n}\left(x, z^{0}, \varepsilon\right), \varepsilon\right), k=1, \cdots, n$;

$8^{\circ}$. The integral

$$
\psi_{j}\left(x_{0}, z^{0}, \varepsilon\right)=\int_{\Gamma_{j x_{0}}} G_{j}\left(x, x_{0}, z^{0}, \varepsilon\right) d x
$$

converges where $\Gamma_{j x_{0}}$ is a curve joining $x^{*}$ on the boundary of $D$ with $x_{0} \in D$ and contained in $D$ except the point $x^{*}$;

$9^{\circ}$. If $x_{0}{ }^{\prime}$ is sufficiently near to $x_{0}$, the relation

$$
\psi_{j}\left(x_{0}, z^{0}, \varepsilon\right)=\int_{\Gamma_{j x_{0}{ }^{\prime}}} G_{j}\left(x, x_{0}, z^{0}, \varepsilon\right) d x+\int_{x_{0^{\prime}}}^{x_{0}} G_{j}\left(x, x_{0}, z^{0}, \varepsilon\right) d x
$$

holds where the integration $\int_{x_{0^{\prime}}}^{x_{0}}$ should be taken along the segment $\overline{x_{0}{ }^{\prime} x_{0}}$.

LEMMA 7. $\psi_{j}\left(x, z_{1}, \cdots, z_{m}, \varepsilon\right), j=1, \cdots, n$, is a solution of the system

$$
\frac{d u_{j}}{d x}=g_{j}\left(x, \phi_{1}\left(x, z^{0}, \varepsilon\right), \cdots, \phi_{n}\left(x, z^{0}, \varepsilon\right)\right), \quad j=1, \cdots, n,
$$

such that $\psi_{j}\left(x^{*}, z^{0}, \varepsilon\right)=0$.

LEMMA 8. If the integral (B) converges uniformly with respect to $z_{1}{ }^{0}$, $\cdots, z_{m}{ }^{0}$ for each $x_{0}$ and $\varepsilon$, then $\psi_{j}(x, z, \varepsilon)$ is holomorphic in the domain $D \times \mathfrak{D}_{m} \times \mathfrak{D}$.

These lemmas were proved by one of the authors when the equalities (A), (B), (C) do not depend upon $\varepsilon$; cf. [4]. Since the discussion goes essentially the same way for our case, we omit the proof here.

4) Proof of the main theorem.

Proposition 1. The mapping $\mathfrak{I}$ transforms $\mathfrak{F}$ into itself. In short, $\mathfrak{I}(\mathfrak{\mho}) \subset \mathfrak{F}$. 
Proof. For the proof, we must show

$$
\left|\bar{\phi}_{j}\left(x_{0}, u^{0}, \varepsilon\right)\right| \leqq K_{N} \max _{k=\beta^{\prime}}^{\beta^{\prime \prime}}\left|u_{k}^{0}\right|^{N / \mu_{k}} \exp \left(-\Re \Lambda_{j}\left(x_{0}, \varepsilon\right)\right)
$$

and

(b) $\bar{\phi}_{j}(x, u, \varepsilon)$ are holomorphic in the domain (6.9).

By (6.7), we have

$$
\left|h_{j}\left(x_{1} U, \phi(x, U, \varepsilon) \exp \Lambda(x, \varepsilon), \varepsilon\right)\right| \leqq\left(A_{j} K_{N}+B_{N}\right) \max _{k=\beta^{\prime}}^{\beta^{\prime \prime}}\left|U_{k}\right|^{N / \mu_{k}} .
$$

Therefore, to show that (a) holds, it suffices to prove

$$
\begin{gathered}
\left|\phi_{j}\left({ }_{\beta} x^{*}, U\left({ }_{\beta} x^{*}, x_{0}, u^{0}, u\right), \varepsilon\right)\right|+\int_{0}^{s_{0}}\left(A_{j} K_{N}+B_{N}\right) \max _{k=\beta^{\prime}}^{\beta^{\prime \prime}}\left|U_{k}\right|^{N / \mu_{k}} \exp \left(-\Re \Lambda_{j}(x, \varepsilon)\right) d s \\
\leqq K_{N} \max _{k=\beta^{\prime}}\left|u_{k}\right|^{N / \mu_{k}} \exp \left(-\Re \Lambda_{j}\left(x_{0}, \varepsilon\right)\right),
\end{gathered}
$$

where $s_{0}$ is the length of the segment ${ }_{\beta} \Gamma_{x_{0}}$. Since the above inequality is trivial when $s_{0}=0$, we have only to show that

$$
\begin{aligned}
&(\left.A_{j} K_{N}+B_{N}\right) \max _{k=\beta^{\prime}}^{\beta^{\prime \prime}}\left|U_{k}\right|^{N / \mu_{k}} \exp \left(-\Re \Lambda_{j}(x, \varepsilon)\right) \leqq K_{N} \frac{d}{d s}\left[\max _{k=\beta^{\prime}}^{\beta^{\prime \prime}}\left|U_{k}\right|^{N / \mu_{k}} \exp \left(-\Re \Lambda_{j}(x, \varepsilon)\right)\right] . \\
& \quad \text { If }\left(\beta^{\prime}, \cdots, \beta^{\prime \prime}\right) \neq\left(n^{\prime}+1, \cdots, n^{\prime \prime}-1\right), \text { we have, by Lemma 6, } \\
& \frac{d}{d s}\left\{\max _{k=\beta^{\prime}}^{\beta^{\prime \prime}}\left|U_{k}\right|^{N / \mu_{k}} \exp \left(-\Re \Lambda_{j}(x, \varepsilon)\right)\right\} \\
&= {\left[-\frac{d}{d s} \Re \Lambda_{j}(x, \varepsilon) \max _{\beta=\beta^{\prime}}^{\beta^{\prime \prime}}\left|U_{k}\right|^{N_{k}}+N\left(\max _{k=\beta^{\prime}}^{\beta^{\prime \prime}}\left|U_{k}\right|^{1 / \mu_{k}}\right)^{N-1} \frac{d}{d s} \max _{k=\beta^{\prime}}^{\beta^{\prime \prime}}\left|U_{k}\right|^{1 / \mu_{k}}\right] \exp \left(-\Re \Lambda_{j}(x, \varepsilon)\right) } \\
&= \max _{k=\beta^{\prime}}\left|U_{k}\right|^{N / \mu_{k}}\left[-\frac{d}{d s} \Re \Lambda_{j}(x, \varepsilon)+\frac{N}{\max _{k=\beta^{\prime}}\left|U_{k}\right|^{1 / \mu_{k}}} \frac{d}{d s} \max _{k=\beta^{\prime}}^{\beta^{\prime \prime}}\left|U_{k}\right|^{1 / \mu_{k}}\right] \exp \left(-\Re \Lambda_{j}(x, \varepsilon)\right) \\
& \geqq \max _{k=\beta^{\prime}}^{\beta^{\prime \prime}}\left|U_{k}\right|^{N / \mu_{k}}\left[-\frac{d}{d s} \Re \Lambda_{j}(x, \varepsilon)+\frac{N \sin \gamma}{4|\varepsilon| \beta^{\sigma^{*}}} \min _{k=\beta^{\prime}}^{\beta^{\prime \prime}} \frac{\mid \lambda_{k}\left(\sigma-\sigma_{k^{\prime}}(0) \mid\right.}{\mu_{k}}\right] \exp \left(-\Re \Lambda_{j}(x, \varepsilon)\right)
\end{aligned}
$$

where

$$
{ }_{\beta} \sigma^{*}=\left\{\begin{aligned}
\sigma_{1}{ }^{*} & \text { if } \quad\left(\beta^{\prime}, \cdots, \beta^{\prime \prime}\right)=\left(1, \cdots, \alpha_{1}\right), \\
\sigma_{m} * & \text { if } \quad\left(\beta^{\prime}, \cdots, \beta^{\prime \prime}\right)=\left(\alpha_{m-1}+1, \cdots, n\right),
\end{aligned}\right.
$$

If $\sigma_{\jmath} \leqq{ }_{\beta} \sigma^{*}$, we have

$$
-\frac{d}{d s} \Re \Lambda_{j}(x, \varepsilon)+\frac{N \sin \gamma}{4|\varepsilon| \beta^{\sigma^{*}}} \min _{k=\beta^{\prime}}^{\beta^{\prime \prime}} \frac{\left|\lambda_{k}^{\left(\sigma-\sigma_{k}\right)}(0)\right|}{\mu_{k}} \geqq \frac{N \sin \gamma}{5|\varepsilon| \beta^{\sigma^{*}}} \min _{k=\beta^{\prime}}^{\beta^{\prime \prime}} \frac{\left|\lambda_{k}^{\left(\sigma-\sigma_{k}\right)}(0)\right|}{\mu_{k}}
$$

by taking $N$ sufficiently large. If $\sigma_{\jmath}>{ }_{\beta} \sigma^{*}$, we have either

$$
j \leqq n^{\prime}, \quad \text { i.e. } \quad\left(\beta^{\prime} \cdots, \beta^{\prime \prime}\right)=\left(\alpha_{m-1}+1, \cdots, n\right),
$$

or

$$
j \geqq n^{\prime \prime}, \quad \text { i.e. } \quad\left(\beta^{\prime}, \cdots, \beta^{\prime \prime}\right)=\left(1, \cdots, \alpha_{1}\right) .
$$

In these cases, ${ }_{\beta} \Gamma_{x_{0}}$ coincides with $\Gamma_{\jmath x_{0}}$ in Lemma 1 . Hence, by Lemma 1 , 


$$
\begin{aligned}
& -\frac{d}{d s} \Re \Lambda_{j}(x, \varepsilon)+\frac{N \sin \gamma}{4|\varepsilon| \beta^{\sigma^{*}}} \min _{k=\beta^{\prime}} \frac{\left|\lambda_{k}{ }^{\left(\sigma-\sigma_{k}\right)}(0)\right|}{\mu_{k}} \\
\geqq & \frac{\left|\lambda_{j}{ }^{\left(\sigma-\sigma_{j}\right)}(0)\right| \sin \gamma}{2|\varepsilon|^{\sigma j}}+\frac{N \sin \gamma}{4|\varepsilon|^{\sigma^{*}}} \min _{k=\beta^{\prime}}^{\beta^{\prime \prime}} \frac{\left|\lambda_{k}^{\left(\sigma-\sigma_{k}\right)}(0)\right|}{\mu_{k}}>\frac{N \sin \gamma}{5|\varepsilon| \beta^{\sigma^{*}}} \min _{k=\beta^{\prime}}^{\beta^{\prime \prime}} \frac{\left|\lambda_{k}{ }^{\left(\sigma-\sigma_{k}\right)}(0)\right|}{\mu_{k}}
\end{aligned}
$$

by taking $N$ sufficiently large. Thus, in any way, (6.13) is implied by the inequality

$$
A_{j} K_{N}+B_{N} \leqq K_{N} \frac{N \sin \gamma}{5|\varepsilon| \beta^{\sigma^{*}}} \min _{k=\beta^{\prime}}^{\beta^{\prime \prime}} \frac{\left|\lambda_{k}^{\left(\sigma-\sigma_{k}\right)}(0)\right|}{\mu_{k}}
$$

which will be realized if $K_{N}$ and $1 / \delta_{0}$ are chosen so large that we have

$$
A_{\jmath}<\frac{N \sin \gamma}{5 \delta_{0}^{\beta^{\sigma^{*}}}} \min _{k=\beta^{\prime}}^{\beta^{\prime \prime}} \frac{\left|\lambda_{k}^{\left(\sigma-\sigma_{k}\right)}(0)\right|}{\mu_{k}} .
$$

To examine the remainig case

$$
\left(\beta^{\prime}, \cdots, \beta^{\prime \prime}\right)=\left(n^{\prime}+1, \cdots, n^{\prime \prime}-1\right),
$$

we must notice that $U_{\jmath}$ does not depend upon $x$ for $j=n^{\prime}+1, \cdots, n^{\prime \prime}-1$. Since, recalling the discussions in section 4 , the functions $p_{j \mathfrak{f}_{\beta}}(x, \varepsilon), j=n^{\prime}+1$, $\cdots, n^{\prime \prime}-1$, can be determined in such a way that they all vanish at $x={ }_{\beta} x^{*}$. Therefore we can suppose that

$$
\phi_{j}\left({ }_{\beta} x^{*}, U\left({ }_{\beta} x^{*}, x_{0}, u^{0}, \varepsilon\right), \varepsilon\right)=0, \quad j=n^{\prime}+1, \cdots, n^{\prime \prime}-1 .
$$

Now, for $\sigma_{\jmath}>0$, it follows from Lemma 1 that

$$
\frac{d}{d s} \exp \left(-\Re \Lambda_{j}(x, \varepsilon)\right) \geqq \frac{\left|\lambda_{j}{ }^{\left(\sigma-\sigma_{j}\right)}(0)\right| \sin \gamma}{2|\varepsilon|^{\sigma_{j}}} \exp \left(-\Re \Lambda_{j}(x, \varepsilon)\right) .
$$

Thus (6.13) follows from the inequality

$$
A_{j} K_{N}+B_{N}<K_{N} \frac{\left|\lambda_{j}{ }^{\left(\sigma-\sigma_{j}\right)}(0)\right| \sin \gamma}{2|\varepsilon|^{\sigma_{\jmath}}}
$$

which can be realized if $\delta_{0}$ is chosen sufficiently small. For $\sigma_{\jmath}=0,(6.12)$ is equivalent to

$$
s_{0}\left(A_{j} K_{N}+B_{N}\right) \max _{k=\beta^{\prime}}^{\beta^{\prime \prime}}\left|U_{k}\right|^{N / \mu_{k}} \leqq K_{N} \max _{k=\beta^{\prime}}^{\beta^{\prime \prime}}\left|U_{k}\right|^{N / \mu_{k}}
$$

because $U_{k}$ is independent of $x$. This can be easily verified by taking $2 \rho\left(\geqq s_{0}\right)$ so small that we have $A_{j} s_{0}<1$.

Thus we have proved (a).

In order to prove (b), we consider following correspondence:

1. the system $(5.2)_{\nu}$ to $(\mathrm{A})$;

2. $h_{j}(x, u, w, \varepsilon) \exp \left(-\Lambda_{\jmath}(x, \varepsilon)\right)$ to $G_{j}$ in $7^{0}$;

3. $\left.\phi_{j}\left(x_{0}, u^{0}, \varepsilon\right)-\phi_{j}\left({ }_{\beta} x^{*}, U\left({ }_{\beta} x^{*}, x_{0}, u^{0}, \varepsilon\right), \varepsilon\right)\right)$ to $\phi_{j}\left(x_{0}, z^{0}, \varepsilon\right)$ in (B);

4. ${ }_{\beta} \Gamma_{x_{0}}$ to $\Gamma_{j x_{0}}^{v}$ in (B).

The inequality (a) just established assures us the uniform convergence of the integral 


$$
\int_{\beta} h_{x_{0}}\left(x_{0}, U\left(x, x_{0}, u^{0}, \varepsilon\right), \phi\left(x, U\left(x, x_{0}, u^{0}, \varepsilon\right) \exp \Lambda(x, \varepsilon) \varepsilon\right), \varepsilon\right) \exp \left(-\Lambda_{j}(x, \varepsilon)\right) d x
$$

which corresponds to

$$
\int_{\Gamma_{j x_{0}}} G_{j}\left(x, x_{0}, z^{0}, \varepsilon\right) d x
$$

in (B) by the above defined correspondence. Therefore the Lemma 8 can be applied and we are immediately led to the condition (b).

Thus we have proved the Proposition 1.

Since, the integral in (6.11) being uniformly convergent, $\mathfrak{I}$ is a continuous mapping of $\mathfrak{F}$ with respect to the topology of uniform convergence (in wide sense), we can conclude, from Proposition 1, the existence of a fixed point of the mapping $\mathfrak{I}$; namely the system $\left(\phi_{1}, \cdots, \phi_{n}\right)$ of $\mathfrak{F}$ such that

$$
\left(\phi_{1}, \cdots, \phi_{n}\right)=\left(\bar{\phi}_{1}, \cdots, \bar{\phi}_{n}\right) \text {. }
$$

Proposition 2. The system $\left(\phi_{1}, \cdots, \phi_{n}\right)$ of $\mathfrak{F}$ corresponding to a fixed point of $\mathfrak{I}$ is a solution of the system (6.8).

Proof. To prove it, we have only to show that, for any $\left(\phi_{1}, \cdots, \phi_{n}\right)$ of $\mathfrak{F}$, $\left(\bar{\phi}_{1}, \cdots, \bar{\phi}_{n}\right)$ is a solution of the system

$$
\frac{d \bar{\phi}_{j}}{d x}=h_{j}(x, U, \phi \exp \Lambda(x, \varepsilon), \varepsilon), \quad j=1, \cdots, n,
$$

which can be easily derived from Lemma 7 .

Thus we have established that there exists, in the family $\mathfrak{F}$, a solution of (6.8). We shall denote it by $\psi_{j N^{\beta}}\left(x, U\left(x, x_{0}, u^{0}, \varepsilon\right), \varepsilon\right)$.

Proposition 3. The solution $\psi_{j N^{\beta}}$ of (6.8) such that

is unique.

$$
\psi_{j N^{\beta}} \beta(x, U, \varepsilon)=o\left(\max _{k=\beta^{\prime}}^{\beta^{\prime \prime}}\left|U_{k}\right|^{N / \mu_{i}} \exp \left(-\Lambda_{j}(x, \varepsilon)\right)\right)
$$

Proof. Assume the contrary, and let $z_{\jmath}, j=1, \cdots, n$, be the difference of any two solutions. Then, evidently, the differential inequality

$$
\frac{d\left|z_{\jmath}\right|}{d s} \leqq A_{\jmath} \max _{k}\left(\left|z_{k}\right| \exp \left(\Re \Lambda_{k}(x, \varepsilon)\right)\right) \exp \left(-\Re \Lambda_{j}(x, \varepsilon)\right)
$$

must hold. If we put

$$
\max _{\beta \Gamma x_{0}, j}\left\{\left.\left|z_{\jmath} \max _{k=\beta^{\prime}}^{\beta^{\prime \prime}}\right| U_{k}\right|^{-N / \mu_{k}} \exp \Lambda_{j}(x, \varepsilon) \mid\right\} \equiv \widetilde{K}>0,
$$

it follows from (6.14) that

$$
\left|z_{\jmath}\right| \leqq \int_{0}^{s_{0}} A_{j} \widetilde{K} \max _{k=\beta^{\prime}}^{\beta^{\prime \prime}}\left|U_{k}\right|^{N / \mu_{k}} \exp \left(-\Re \Lambda_{j}(x, \varepsilon)\right) d s .
$$

As we have already seen in the proof of the Proposition 1, we have 


$$
\int_{0}^{s_{0}} A_{j} \widetilde{K} \max _{k=\beta^{\prime}}^{\beta^{\prime \prime}}\left|U_{k}\right|^{N / \mu_{k}} \exp \left(-\Re \Lambda_{j}(x, \varepsilon)\right) d s \leqq \kappa_{j} \max _{k=\beta^{\prime}}^{\beta^{\prime \prime}}\left|U_{k}\right|^{N / \mu_{k}} \exp \left(-\Re \Lambda_{j}(x, \varepsilon)\right)
$$

where

$$
\kappa_{\jmath}= \begin{cases}5|\varepsilon| \beta^{\sigma^{*}} /\left(N \sin \gamma \cdot \min _{k=\beta^{\prime}} \frac{\left|\lambda_{k}{ }^{\left(\sigma-\sigma_{k}\right)}(0)\right|}{\mu_{k}}\right),\left(\beta^{\prime}, \cdots, \beta^{\prime \prime}\right)=\left(1, \cdots, \alpha_{1}\right),\left(\alpha_{m-1}+1, \cdots, n\right), \\ 2|\varepsilon|^{\sigma_{\jmath}} /\left(\left|\lambda_{\jmath}{ }^{\left(\sigma-\sigma_{j}\right)}(0)\right| \sin \gamma,\right) & \left(\beta^{\prime}, \cdots, \beta^{\prime \prime}\right)=\left(n^{\prime}+1, \cdots, n^{\prime \prime}-1\right), \quad \sigma_{\jmath}>0, \\ s_{0}(\leqq 2 \rho), & \left(\beta^{\prime}, \cdots, \beta^{\prime \prime}\right)=\left(n^{\prime}+1, \cdots, n^{\prime \prime}-1\right), \quad \sigma_{\jmath}=0 .\end{cases}
$$

Therefore, if we choose $\delta_{0}$ and $2 \rho$ so small that we have $\kappa_{\jmath} A_{\jmath}<1$, we obtain $\widetilde{K}<\widetilde{K}$ which is a contradiction. Hence $z \equiv 0$ and the unicity of the solution is established.

Now consider the solution of the system (1.1):

$$
S_{j N}^{\beta}(x, U, \varepsilon)=Q_{j N}^{\beta}(x, U, \varepsilon)+\psi_{j N} \beta(x, U, \varepsilon) \exp \Lambda_{j}(x, \varepsilon) .
$$

To complete the proof of the Theorem 4, it remains for us to show that the solution $S_{j N^{\beta}}$ is independent of $N$. Since

$$
\left[Q_{j N^{\prime}}{ }^{\beta}+{\psi_{j N^{\prime}}}^{\beta} \exp \Lambda_{\jmath}-Q_{j N^{\beta}}{ }^{\beta}\right] \exp \left(-\Lambda_{j}\right), \quad j=1, \cdots, n, \quad N^{\prime}>N,
$$

is a solution of (6.8) satisfying the condition of Proposition 3, it must be equal to $\psi_{j N}{ }^{\beta}$ from the above established unicity. Therefore we have $S_{j N^{\prime}}{ }^{\beta} \equiv S_{j N^{\beta}}{ }^{\beta}$ which shows that $S_{j N}{ }^{\beta}$ is independent of $N$.

Thus we have completed the proof of our main theorem.

7. Concluding remark. We have thus proved the convergence of the formal solutions $\left(\mathrm{F}_{1}\right),\left(\mathrm{F}_{h+1}\right)$ and $\left(\mathrm{F}_{m}\right)$. As we have seen, there exist another formal solutions $\left(F_{\nu}\right), \nu \neq 1, h+1, m$. As for these formal solutions, it is doubtful whether they converge.

If all of $\sigma_{\jmath}$ are equal to $\sigma$ and all of $\lambda_{\jmath}{ }^{\left(\sigma-\sigma_{j}\right)}(0)(\neq 0)$ lie on one side of the straight line passing through the origin on the complex plane, we can construct the general solution of the system (1.1) by Theorem 4. In this case, moreover, it can be shown that we can dispense with the diagonalization of the terms whose degrees with respect to $\varepsilon$ are less than $\sigma$ in the linear part of the second member of (1.1). This has been already shown by Sibuya in his paper cited before.

In conclusion, we wish to express our cordial thanks to Dr. Sibuya by whose work this paper was greatly inspired.

\section{REFERENCES}

[1] HukuhaRA, M., Intégration formelle d'un système d'équations différentielles non linéaires dans le voisinage d'un point singulier. Ann. Mat. Pura Appl. 19 (1940), 35-44.

[2] Hukuhara, M., Sur les propriétés asymptotiques des solutions d'un système d'équations différentielles linéaires contenant un paramètre. Mem. Fac. Sci. Kyushu Imp. Univ. 8 (1937), 249-280. 
[ 3 ] Hukuhara, M., Renzokuna Kansû no Zoku to Syazô. Mem. Fac. Sci. Kyushu Univ. 5 (1950), 61-63.

[4] Iwano, M., Intégration analytique d'un système d'équations différentielles non linéaires dans le voisinage d'un point singulier, I; II. Ann. Mat. Pura Appl. 44 (1957), 261-292; 47 (1959), 91-150.

[5] SibuYA, Y., UCLA dissertation.

\section{ERRATA}

We profit this opportunity to correct some errors in Iwano's former paper cited before.

Iwano [4], I, p. 276: The second member of (19.3) should be replaced by the following expression:

$$
\begin{aligned}
& {\left[\lambda_{j}(x)\left(P_{j N}(x, z)-\delta_{j} z_{j}\right)-\sum_{k=1}^{\alpha_{1}} \lambda_{k}(x) z_{k}\left\{\frac{\partial P_{j N}(x, z)}{\partial z_{k}}-\delta_{j k}\right\}-x^{\sigma+1} \frac{\partial P_{j N}(x, z)}{\partial x}\right] } \\
+ & {\left[x^{\sigma} h_{j}\left(x, P_{N}(x, z)+v\right)\right]+\left[\delta_{j} \lambda_{j}(x) z_{j}-\sum_{k=1}^{\alpha_{1}} \delta_{j k} x^{\sigma+1} \frac{d z_{k}}{d x}\right] } \\
- & {\left[\sum_{k=1}^{\alpha_{1}}\left(x^{\sigma+1} \frac{d z_{k}}{d x}-\lambda_{k}(x) z_{k}\right)\left(\frac{\partial P_{j N}(x, z)}{\partial z_{k}}-\delta_{j k}\right)\right] . }
\end{aligned}
$$

Iwano [4], II, p. 99: The second member of $\left(33.1^{\prime}\right)$ should be amended as follows:

$$
\begin{aligned}
& {\left[\lambda_{j}(x)\left(P_{j N}(x, z)-\delta_{j} z_{j}\right)-\sum_{k=\alpha^{\prime}}^{\alpha^{\prime \prime}} \lambda_{k}(x) z_{k}\left\{\frac{\partial P_{j N}(x, z)}{\partial z_{k}}-\delta_{j k}\right\}-x^{\sigma+1} \frac{\partial P_{j N}(x, z)}{\partial x}\right] } \\
+ & {\left[x^{\sigma} h_{j}\left(x, P_{N}(x, z)+v\right)\right]+\left[\delta_{j} \lambda_{j}(x) z_{j}-\sum_{k=\alpha^{\prime}}^{\alpha^{\prime \prime}} \delta_{j k} x^{\sigma+1} \frac{d z_{k}}{d x}\right] } \\
- & {\left[\sum_{k=\alpha^{\prime}}^{\alpha^{\prime \prime}}\left(x^{\sigma+1} \frac{d z_{k}}{d x}-\lambda_{k}(x) z_{k}\right)\left(\frac{\partial P_{j N}(x, z)}{\partial z_{k}}-\delta_{j k}\right)\right] . }
\end{aligned}
$$

Iwano [4], II, p. 120: The linear differential equation (46.2) and (46.4) should be replaced by

$$
\begin{aligned}
& x^{\sigma+1} \frac{d P_{j \mathfrak{p}^{\prime}}}{d x}=\left(\lambda_{j \mathfrak{p}^{\prime}}(x)+\lambda_{j \mathfrak{p}^{\prime} \sigma} x^{\sigma}\right) P_{j \mathfrak{p}^{\prime}}+x^{\sigma+1} \sum_{h=1}^{n} \Phi_{j h}\left(x, Z_{\alpha^{\prime}}, \cdots, Z_{\alpha^{\prime \prime}}\right) P_{h_{\mathfrak{p}^{\prime}}} \\
& +x^{\sigma} Q_{\mathfrak{p}^{\prime}}\left(x, Z_{\alpha^{\prime}}, \cdots, Z_{\alpha^{\prime \prime}}\right) \text {, } \\
& \Phi_{j h}\left(x, Z_{\alpha^{\prime}}, \cdots, Z_{\alpha^{\prime \prime}}\right) \equiv \sum a_{j k_{1}} \cdots k_{n}(x) \Phi_{1}^{k_{1}} \ldots \Phi_{h-1}{ }^{k_{h-1}} \cdot k_{h} \Phi_{h^{h}}{ }^{k_{h}-1} \cdot \Phi_{h+1}{ }^{k_{h+1}} \ldots \Phi_{n}{ }^{k_{n}} \\
& (j=1, \cdots n),
\end{aligned}
$$

and

$$
\begin{array}{r}
x \frac{d V_{j \mathfrak{p}^{\prime}}}{d x}=\left[x \sum_{h=1}^{n} \Phi_{j h}\left(x, x_{0}, z_{\alpha^{\prime}}^{0}, \cdots, z^{0}{ }_{\alpha^{\prime \prime}}\right) x^{\lambda_{h \mathfrak{p}^{\prime} \sigma}} V_{h \mathfrak{p}^{\prime}} \exp \Lambda_{h \mathfrak{p}^{\prime}}(x)\right. \\
\left.\quad+Q_{j \mathfrak{p}}\left(x, x_{0}, z^{0}{ }_{\alpha^{\prime}}, \cdots, z_{\alpha^{\prime \prime}}^{0}\right)\right] x^{-\lambda_{j \mathfrak{p}^{\prime} \sigma}} \exp \left(-\Lambda_{\jmath \mathfrak{p}^{\prime}}(x)\right), \\
\Phi_{j h}\left(x, x_{0}, z_{\alpha^{\prime}}^{0} \cdots, z^{0}{ }_{\alpha^{\prime \prime}}\right) \equiv \Phi_{j h}\left(x, Z_{\alpha^{\prime}}\left(x, x_{0}, z^{0}{ }_{\alpha^{\prime}}\right), \cdots, Z_{\alpha^{\prime \prime}}\left(x, x_{0}, z^{0}{ }_{\alpha^{\prime \prime}}\right)\right) \\
\quad(j=1, \cdots, n),
\end{array}
$$

respectively. 
Iwano [4], II, p. 122: The argument from 4-th to 12-th line should be corrected as follows:

Cela posé, dans tous les cas, les fonctions $V_{j \mathfrak{p}^{\prime}}\left(x, x_{0}, z^{0}\right)(j=1, \cdots, n)$ se déterminent d'une seule manière par les conditions $V_{j p^{\prime}}\left(0, x_{0}, z^{0}\right)=0$ de sorte que les fonctions $V_{j \mathfrak{p}^{\prime}}(x, z) \equiv V_{j \mathfrak{p}^{\prime}}(x, x, z)$ soient holomorphes dans le domaine (46.1) et représentent les solutions des équations différentielles linéaires (46.4) si l'on y remplace $z$ par $Z$.

En effet, si on porte la solution formelle (45.1), où l'on pose $z=Z$, dans les équations différentielles linéaires (46.2), on aura les équations différentielles linéaires pour déterminer les coefficients $P_{\jmath \mathfrak{p \mathfrak { p } ^ { \prime }}}(x)$. On verra alors que les fonctions $P_{j p \mathfrak{p}^{\prime}}(x)$ sont déterminées demanière qu'elles soient holomorphes et développables asymptotiquement en les séries (45.2) pour le domaines $\mathfrak{D}\left[\Theta^{*}{ }_{\nu \mu_{-}}, \Theta^{*_{\nu \mu_{+}}}, \gamma\right]$. $\mathrm{Si}$, ensuite, on applique les raisonnements tout à fait analogues aux ceux des $\mathrm{n}^{\text {os }} 33,34,35,41$ au système des équations différentielles linéaires (46.4), on pourra démontrer l'existence des fonctions $V_{j \mathfrak{p}^{\prime}}\left(x, x_{0}, z^{0}\right)$.

L'existence des fonctions $V_{j p^{\prime}}(x, z)$ nous montre que les séries (45.1) sont uniformément convergentes pour les valeurs $x, z$ telles qu'elles appartiennent au domaine (46.1).

Department of Mathematics, TOKYo METROPOLITAN UNIVERSITY. 